\title{
Diffeomorphism covariant representations of the holonomy-flux $\star$-algebra
}

\author{
Andrzej Okołów ${ }^{1 *}$ and Jerzy Lewandowski ${ }^{2,1,3 \dagger}$
}

February 14, 2003

\author{
1. Instytut Fizyki Teoretycznej, Uniwersytet Warszawski, ul. Hoża 69, \\ 00-681 Warsaw, Poland \\ 2. Center for Gravitational Physics and Geometry, Physics Department, \\ 104 Davey, Penn State, University Park, PA 16802, USA \\ 3. Max-Planck-Institut für Gravitationsphysik, Albert-Einstein-Institut, \\ 14476 Golm, Germany \\ *oko@fuw.edu.pl \\ $\dagger$ lewand@fuw.edu.pl
}

\begin{abstract}
Recently, Sahlmann [1] proposed a new, algebraic point of view on the loop quantization. He brought up the issue of a $\star$-algebra underlying that framework, studied the algebra consisting of the fluxes and holonomies and characterized its representations. We define the diffeomorphism covariance of a representation of the Sahlmann algebra and study the diffeomorphism covariant representations. We prove they are all given by Sahlmann's decomposition into the cyclic representations of the sub-algebra of the holonomies by using a single state only. The state corresponds to the natural measure defined on the space of the generalized connections. This result is a generalization of Sahlmann's result 2] concerning the $U(1)$ case.
\end{abstract}

\section{Introduction}

The quantum holonomy operators and the quantum flux operators are the basic elements of the quantum geometry [4, 5, 7, 8, 9, and loop quantum gravity [10], the theory also called the Quantum Spin Dynamics (QSD) after the title of Thiemann's series of works [11. Quantum geometry is a 
quantum theory of geometry of initial data of Einstein's theory in terms of the Ashtekar variables. The flux quantum operators correspond to the intrinsic geometry (to the fluxes of the classical triads) whereas the holonomy operators to the extrinsic curvature (the Riemannian connection is combined with the extrinsic curvature into a new $S U(2)$-connection 1-form). In the standard representation, the operators are defined in the Hilbert space given by the natural diffeomorfism invariant measure denoted in this paper by $\mu_{\mathrm{AL}}$ [12] defined on the Ashtekar-Isham quantum configuration space of generalized connections [14, 12, 4]. Every holonomy operator is just the multiplication by the corresponding holonomy variable. Each flux operator is $i$ times certain derivation defined on the differentiable functions of the holonomies [7. These flux operators are self-adjoint. All the basic operators of the quantum geometry (volume, area, length), the scalar constraint as well as the quantum Hamiltonian operators of matter coupled with quantum geometry [11] are constructed by using those elementary holonomy-flux operators [7, 8]. The framework can be also generalized to a loop quantization of diffeomorphism invariant theories of $G$-connections defined over a $d$-dimensional manifold [10]. Recently, Sahlmann [1] proposed a new, more algebraic point of view. It opens the door to a representation theory of the quantum geometry. The main idea is to spell out a definition of a $\star$ algebra constructed from the holonomies and fluxes, that underlies all the loop quantum gravity framework, and to study its representations. The conclusion is that, upon some quite natural assumptions, every representation of the Sahlmann algebra can be characterized by using the direct sum of cyclic representations of the Ashtekar-Isham commutative $C^{\star}$-algebra of the holonomies. Each cyclic representation is defined in a Hilbert space given by a measure on the space of the generalized connections. The main result of Sahlmann is establishing a general form in which the flux operators can be represented. Each flux operator is given by the corresponding derivation plus a suitable multiplication operator (we often call it a correction term). Finally, in the case of the structure group $U(1)$ of the theory, Sahlmann proved that every diffeomorphism covariant representation necessarily corresponds to the natural measure mentioned above [2]

In our paper we introduce a precise definition the holonomy-flux *algebra in the way following from [1]. We name it the Sahlmann algebra. According to the definition, the commutative subalgebra of the holonomies contained in the Sahlmann algebra is not the entire Ashtekar-Isham $C^{\star}$-algebra. Therefore a priori, the representation theory of commutative $C^{\star}$-algebras does not apply. We fill that gap, and show that every representation of the subalgebra extends uniquely to a representation of the entire $C^{\star}$-algebra of 
the holonomies. In this way me merge Sahlmann's characterization of the holonomy-flux $\star$-algebra. Up to now, diffeomorphism invariance and covariance are not well defined notions in the context of representations. To see why this is so, first observe that the action of (analytic) diffeomorphisms in the Sahlmann algebra induces an action on the representations. We do not expect a representation to be invariant, but it is not even clear how to define covariance of a representation. This is because diffeomorphisms do not act naturally in the carrier space of a given representation (Sahlmann's characterization depends on a choice of the cyclic vectors). We propose a solution of that issue and formulate a definition of a representation of the Sahlmann algebra covariant with respect to the diffeomorphisms. On a working level, our definition coincides with the one used by Sahlmann in the $U(1)$ case mentioned above. Our main result is a proof that every diffeomorphism covariant representation of the holonomy-flux $\star$-algebra, upon an assumption about the domain, is given by Sahlmann's characterization such that all the measures used in the direct sum are the same natural measure $\mu_{\mathrm{AL}}$. This result is valid in the case of the covariance with respect to the group of the analytic diffeomorphisms of $\Sigma=\mathbb{R}^{d}(d \geq 2)$, and for an arbitrary compact, connected Lie group. The only remaining ambiguity is in the definition of the representation of the flux operators in the correction term. However, the identification of the carrier Hilbert space as an orthogonal product of the spaces given by the natural measure $\mu_{\mathrm{AL}}$ enables one to use the powerful machinery of the decompositions into the spin-network functions. It can be used to reduce considerably the remaining freedom in the representation of the quantum fluxes. One more separate result of our paper is that the only cyclic representation of the holonomy-flux $\star$-algebra $\mathfrak{A}$ given by the identity map is the one given by the natural measure (see Lemma 6.2).

The motivation for our work came from numerous discussions with Hanno Sahlmann about his published and unpublished results. For an independent account on the diffeomorphism covariant representations of the holonomy-flux-algebra see 3 .

\section{Preliminaries}

The loop quantization framework was introduced and developed in [4]-14]. The goal of the program is the canonical quantization of diffeomorphism invariant and gauge invariant theories of connections. The main application is loop quantum gravity. Then, in the $3+1$ case, the phase space is the cotangent bundle of the space of $S U(2)$-connections defined on a (trivial) 
bundle over a 3-dimensional manifold. In this paper we deal with the arbitrarily dimensional case. For the simplicity we consider $G$-connections, where $G$ is a compact, connected Lie group, defined on a trivial bundle over a $d$-dimensional manifold $\Sigma$. The generalization to a non-trivial bundle is also possible [13, 4]. The framework considered in this paper applies to any theory, whose phase space $\mathcal{A} \times \mathcal{E}$ consists of pairs of canonically conjugate fields $(A, \tilde{E})$ defined on $\Sigma$, where: $A$ is a Lie algebra $G^{\prime}$ valued differential 1 -form and $\tilde{E}$ is a vector density of weight 1 which takes values in the space $G^{*}$ dual to the Lie algebra $G^{\prime}$. That is, the Poisson bracket between two functions $H$ and $L$ defined on $\mathcal{A} \times \mathcal{E}$ is given by the standard relation:

$$
\{H, L\}=\int_{\Sigma} d x\left(\frac{\delta H}{\delta A_{a}^{i}(x)} \frac{\delta L}{\delta \tilde{E}_{i}^{a}(x)}-\frac{\delta L}{\delta A_{a}^{i}(x)} \frac{\delta H}{\delta \tilde{E}_{i}^{a}(x)}\right),
$$

where the fields $A$ and $\tilde{E}$ are expressed by their components with respect to a basis $\left(\tau_{i}\right), i=1, \ldots, n$ of the Lie algebra $G^{\prime}$, the dual to $\left(\tau_{i}\right)$ basis $\left(\tau^{* i}\right)$ of $G^{*}$, and a (local) coordinate chart $\left(x^{a}\right), a=1, \ldots, d$ on $\Sigma$, i.e.:

$$
A=A_{a}^{i} \tau_{i} \otimes d x^{a} \quad \tilde{E}=\tilde{E}_{i}^{a} \tau^{* i} \otimes \partial_{a} .
$$

The integrand is a density of the weight 1 , hence the integral does not depend on choice of a coordinate chard $\left(x^{a}\right)$. Every map $a: \Sigma \rightarrow G$ defines a gauge transformation:

$$
(A, \tilde{E}) \mapsto\left(a^{-1} A a+a^{-1} d a, a^{-1} \tilde{E} a\right)
$$

where, being more formal, given $v \in G^{\prime}$, the element $a^{-1} v a$ is obtained by the adjoint action $\operatorname{ad}\left(a^{-1}\right) v$ of $G$ in $G^{\prime}$, and the $G^{\prime}$ valued differential 1form $a^{-1} d a$ is the pullback $a^{*} \theta_{\mathrm{MC}}$ of the Maurer-Cartan form $\theta_{\mathrm{MC}}$. Those transformations are often called "the Yang-Mills" gauge transformations, to emphasize that the group of all the gauge transformations of the theory (in the sense of the hamiltonian approach) is bigger and contains also the diffeomorphisms.

The fields $A \in \mathcal{A}$ and $\tilde{E} \in \mathcal{E}$ are referred to as connections and, respectively, electric fields. In the loop quantization the space $\mathcal{A}$ of connections is chosen to be a configuration space and $\mathcal{E}$ becomes the space of momenta. In this paper we do not consider any other fields coupled to $(A, \tilde{E})$, however, in the presence of other fields the framework presented still applies to the $(A, \tilde{E})$ degrees of freedom and there is known a natural extension of this approach which accommodates the coupled fields [1]. 


\subsection{The holonomy-flux variables}

The first step in the loop quantization is a non-standard choice of elementary classical variables that will have direct quantum analogs. They are the functions of finitely many holonomies along curves in $\Sigma$ - the cylindrical functions - and fluxes of the electric field across $(d-1)$-dimensional submanifolds in $\Sigma$. We recall now the definitions in detail.

Consider an oriented 1-dimensional submanifold $e$ with boundary given by an embedding of an interval: $e:\left[t_{0}, t_{1}\right] \rightarrow \Sigma$. It is called an edge. The holonomy transport $h_{e}\left(t, t_{0}, A\right)$ along an the edge $e$ from $e\left(t_{0}\right)$ to $e\left(t_{1}\right)$ with respect to a connection $A$ is an element of $G$ defined by the following differential equation and initial conditon:

$$
\begin{aligned}
\frac{d}{d t} h_{e}\left(t, t_{0}, A\right) & =-A_{a}(e(t)) \dot{e}^{a}(t) h_{A, e}\left(t, t_{0}\right), \\
h_{e}\left(t_{0}, t_{0}, A\right) & =\mathbb{I}
\end{aligned}
$$

where $\mathbb{I} \in G$ stands for the neutral element of $G$. Given $A \in \mathcal{A}$, the parallel transport along an edge $e$ is denoted by:

$$
A(e):=h_{e}\left(t_{1}, t_{0}, A\right)=\mathcal{P} \exp \left(-\int_{e} A\right)
$$

and called the holonomy variable. The holonomy variable $A(e)$ is insensitive on orientation preserving reparametrizations of $e$. Denote the splitting of an edge $e$ into two edges $e_{1}, e_{2}$ of the same orientation by $e=e_{2} \circ e_{1}$, and the edge obtained by reversing the orientation of $e$ by $e^{-1}$. The holonomy variable satisfies the following relations:

$$
A\left(e_{2}\right) A\left(e_{1}\right)=A\left(e_{2} \circ e_{1}\right), A\left(e^{-1}\right)=A(e)^{-1} .
$$

Definition 2.1 A complex valued function $\Psi$ defined on $\mathcal{A}$ is called cylindrical, if there is a finite set of edges $\left\{e_{1}, \ldots, e_{N}\right\}$ and a function $\psi$ defined on $G^{N}$ such that:

$$
\Psi(A)=\psi\left(A\left(e_{1}\right), \ldots, A\left(e_{N}\right)\right)
$$

We say $\Psi$ is compatible with the set of edges $\left\{e_{1}, \ldots, e_{N}\right\}$.

We consider in this work the set $\mathrm{Cyl}^{\infty}$ of all the cylindrical functions given by all the $C^{\infty}$ functions $\psi$ in (2.4) (analogously, we define the set $\mathrm{Cyl}^{0}$ 
of all the cylindrical functions given by all the continuous functions $\psi$ ) . $\mathrm{Cyl}^{\infty}$ is a subalgebra of the $\star$-algebra of all the complex-valued functions defined on $\mathcal{A}$ (with $\star$ being the complex conjugation). A naturally defined norm in $\mathrm{Cyl}^{\infty}$ is the sup norm,

$$
\|\psi\|_{\text {sup }}:=\sup _{A \in \mathcal{A}}|\Psi(A)| .
$$

The completion $\overline{\mathrm{Cyl}^{\infty}}$ in that norm together with $\star$ and the norm is the Ashtekar-Isham $C^{\star}$-algebra denoted by Cyl below. The Gel'fand-Naimark spectrum of that algebra is characterized as the space $\overline{\mathcal{A}}$ of the generalized connections being homomorphisms between the groupoid of the curves in $\Sigma$ and the gauge group $G$ [4] (see also Section 4.2).

To define the flux of $E$ fix: $(i)$ a finite $(d-1)$ submanifold $S \subset \Sigma(i i)$ an orientation in the normal bundle to $S$, and (iii) a function $f: S \rightarrow G^{\prime}$.

The corresponding flux of $\tilde{E}$ is defined to $b^{1}$

$$
E(S, f):=\frac{1}{(d-1) !} \int_{S} \tilde{E}_{i}^{a} f^{i} \epsilon_{a a_{1} \ldots a_{d-1}} d x^{a_{1}} \wedge \ldots \wedge d x^{a_{d-1}},
$$

where the (local) orientation of $S$ used in the integral is determined by the orientation of the normal bundle and the orientation of a given local coordinate chart.

\subsection{The Ashtekar-Corichi-Zapata Poisson algebra}

Now we turn to the Poisson bracket relations between the elementary classical variables. It should be emphasized, however, that the fluxes and the cylindrical functions are in fact distributional objects from the point of view of the Poisson bracket (2.1). Therefore, to apply the Poisson bracket (2.1) to the fluxes and holonomies some extension procedure is needed. Ashtekar, Corichi and Zapata 9] proposed a very natural passage. They use the observation that every function defined on the phase space $\mathcal{A} \times \mathcal{E}$ and linear in $\tilde{E}$ defines a derivation $X$ in the algebra of functions defined on $\mathcal{A}$, namely

$$
\left\{\Phi, P_{X}\right\}=: X \Phi
$$

where the function linear in $\tilde{E}$ is denoted by $P_{X}$. Then, for functions $\Phi, \Phi^{\prime}$ independent of $\tilde{E}$, and any two functions $P_{X}, P_{X^{\prime}}$ linear in $\tilde{E}$, the following relations are true,

$$
\left\{P_{X}, P_{X^{\prime}}\right\}=P_{\left[X, X^{\prime}\right]}, \quad\left\{\Phi, \Phi^{\prime}\right\}=0 .
$$

\footnotetext{
${ }^{1} \epsilon_{a a_{1} \ldots a_{d-1}}$ is a Levi-Civita density of weight -1 on $\Sigma$.
} 
Therefore, assigning to each of the linear functions $P_{X}$ the derivation $X$, and to each of the $\tilde{E}$ independent functions $\Phi$ the multiplication operator by the given function $\Phi$ defines an isomorphism between: a) the Poisson algebra spanned by the functions $P_{X}$ and $\Phi$, and $b$ ) the Lie algebra of operators spanned by the derivations $X$, and the multiplication by the functions $\Phi$ operators. The isomorphism maps $\{,\} \mapsto[$,$] . The idea of [9] is to define an$ algebra of the classical elementary variables from the cylindrical functions and the flux variables as a Lie algebra of operators analogous to the one described above. Indeed, as we explain below, every electric flux does define through the Poisson bracket (2.5) a non-trivial derivation operator in the algebra $\mathrm{Cyl}^{\infty}$. We start from the discussion of that operator.

For the Poisson bracket (2.5) between a cylindrical function $\Psi$ compatible with a set of edges $\left\{e_{1}, \ldots, e_{N}\right\}$ and the flux $E(S, f)$ across a submanifold $S$ to be finite, the intersection between the edges $\left\{e_{1}, \ldots, e_{N}\right\}$ and $S$ has to satisfy some regularity property. To ensure that property arbitrarily, we fix an analytic structure on $\Sigma$ and define regular analytic submanifolds in the following way: $(i)$ 0-dimensional regular analytic sumbanifold of $\Sigma$ is a one-element subset of $\Sigma($ ii $)$ an $m$-dimensional $(0<m<\operatorname{dim} \Sigma)$ regular analytic submanifold $M$ is an analytic orientable $m$-submanifold such that its closure $\bar{M}$ is a compact subset of an analytic $m$-dimensional submanifold of $\Sigma$ and $\bar{M} \backslash M$ is a finite sum of regular analytic submanifolds of the lower dimensions. We assume that all the edges used in the definition of the cylindrical functions are the closures of the regular analytic 1-submanifolds. We also assume that the family of all the submanifolds $S$ used in the flux definition contains only regular analytic $(d-1)$-submanifolds (later, this family will be extended to a larger one by set differences and intersections of the regular analytic $S$ 's).

Upon the regular analyticity assumption, every cylindrical function on $\mathcal{A}$ is compatible with an analytically embedded graph, whose definition we recall below.

Definition 2.2 An analytically embedded graph $\gamma$ in $\Sigma$ is a set of analytic edges $\left\{e_{1}, \ldots, e_{N}\right\}$ in $\Sigma$ such that two distinct edges can meet each other only in their endpoints. The edges will be called the edges of the graph $\gamma$, and their endpoints the vertices of $\gamma$.

To calculate the Poisson bracket between a given cylindrical function $\Psi \in \mathrm{Cyl}^{\infty}$ and the flux function $E(S, f)$ across a surface $S$, we select a graph compatible with $\Psi$ appropriately. Given $\Psi$ and $S$ we choose a graph $\gamma$ compatible with $\Psi$ such that every edge of $\gamma$ either: $(i)$ is contained in $S$ 
(modulo its end points), or (ii) intersects $S$ at exactly one endpoint, or (iii) does not intersect $\gamma$ at all. The orientation in the normal bundle $S$ is used to distinguish the subset of the class ( $i i)$ edges placed 'up' ('down') the surface $S$. Those edges will be labelled by $I_{\text {up }}\left(I_{\text {down }}\right)$. After the substitution of $\Psi$ and $E(S, f)$ for the functions $H$ and $L$ in (2.1) the direct calculation gives the following result,

$$
\begin{array}{r}
\{\Psi, E(S, f)\}=\frac{1}{2} \sum_{v \in S} f^{i}(v)\left(\sum_{e_{I_{\text {down }}}} X_{i, v, e_{I_{\mathrm{down}}}}-\sum_{e_{I_{\mathrm{up}}}} X_{i, v, e_{I_{\mathrm{up}}}}\right) \Psi=: \\
=: X_{S, f} \Psi
\end{array}
$$

where given $v, e_{I_{\mathrm{up} / \mathrm{down}}}$ runs through the subset of the edges of $\gamma$ such that $v$ is an isolated intersection between $e_{I_{\text {up } / \text { down }}}$ and $S$, and

$$
\begin{aligned}
\left(X_{i, v, e_{I}} \Psi\right)\left(A\left(e_{1}\right), \ldots, A\left(e_{I}\right), \ldots, A\left(e_{N}\right)\right):= \\
\quad:= \begin{cases}\left.\frac{d}{d s}\right|_{s=0} \Psi\left(\ldots, A\left(e_{I}\right) \exp \left(s \tau_{i}\right), \ldots\right), & \text { if } v \text { is the beginning of } e_{I} \\
\left.\frac{d}{d s}\right|_{s=0} \Psi\left(\ldots, \exp \left(-s \tau_{i}\right) A\left(e_{I}\right), \ldots\right), & \text { if } v \text { is the end of } e_{I}\end{cases}
\end{aligned}
$$

In conclusion, each electric field flux $E(S, f)$ corresponds to the derivation $X_{S, f}: \mathrm{Cyl}^{\infty} \rightarrow \mathrm{Cyl}^{\infty}$. With this result we go back to the construction of the algebra of the classical elementary observables from the cylindrical functions and fluxes.

Consider the complex vector space spanned by the operators:

$$
\begin{gathered}
\mathrm{Cyl}^{\infty} \ni \Psi^{\prime} \mapsto \Psi \Psi^{\prime} \in \mathrm{Cyl}^{\infty}, \\
\mathrm{Cyl}^{\infty} \ni \Psi^{\prime} \mapsto X_{S, f} \Psi^{\prime} \in \mathrm{Cyl}^{\infty}
\end{gathered}
$$

given by all the cylindrical functions $\Psi \in \mathrm{Cyl}^{\infty}$ and all the fluxes $E(S, f)$ corresponding to all submanifolds $S$ and smearing functions $f$. Extend the resulting space of operators to the (smallest possible) operator Lie algebra, and denote it by $\mathfrak{A}_{\mathrm{ACZ}}$.

Definition 2.3 The algebra $\left(\mathfrak{A}_{\mathrm{ACZ}},\{\}=,[],\right)$ is the Ashtekar-Corichi-Zapata algebra of the elementary classical variables whose classical bracket $\{$, is by definition the commutator (no $i$ factor thus far) between the derivation and/or multiplication operators.

The Ashtekar-Corichi-Zapata algebra can be written as the direct sum

$$
\mathfrak{A}_{\mathrm{ACZ}}=\mathrm{Cyl}^{\infty} \oplus \mathcal{X},
$$


where $\mathcal{X}$ are the derivations obtained by taking all the $X_{S, f}$ operators given by all the submanifolds $S$ and functions $f$ and all the (multiple) commutators.

The derivation operator $X_{S, f}$ was defined for a $(d-1)$-dimensional submanifold $S$. However, the definition (2.7) of the operator is naturally extended to $(d-1)$-dimensional manifold with boundary embedded in $\Sigma$. Indeed, consider two operators $X_{S, f}$ and $X_{S^{\prime}, f^{\prime}}$ such that $S^{\prime} \subset S$ and $f_{\mid S^{\prime}}=f^{\prime}$. Then the operator

$$
X_{S \backslash S^{\prime}, f}:=X_{S, f}-X_{S^{\prime}, f^{\prime}}
$$

is an operator of a flux across $S \backslash S^{\prime}$ being an embedded manifold with boundary.

A (classical) anomaly in the Ashtekar-Corichi-Zapata algebra is that a single classical elementary variable may correspond to two different derivations. An example is a surface $S$ split by an edge $e$ into two pieces, $S=$ $S_{1} \cup e \cup S_{2}$. Then, $E(S, f)=E\left(S_{1}, f\right)+S\left(S_{2}, f\right)$, but $X_{S, f} \neq X_{S_{1}, f}+X_{S_{2}, f}$. Another kind of anomaly are the commutators $\left[X_{S_{1}, f_{1}}, X_{S_{2}, f_{2}}\right]$ when $S_{1} \cap S_{2}$ is 1-dimensional.

\section{The Sahlmann holonomy-flux $\star$-algebra}

The quantization consists in assigning to every elementary classical variable, that is to every element of the Ashtekar-Corichi-Zapata Lie algebra $\left(\mathfrak{A}_{\mathrm{ACZ}},\{\},\right)$, an element of an appropriate $\star$-algebra $(\mathfrak{A}, \star)$,

$$
\text { ^: } \mathfrak{A}_{\mathrm{ACZ}} \rightarrow \mathfrak{A},
$$

such that

$$
\widehat{\{a, b\}}=i[\hat{a}, \hat{b}] \text {. }
$$

In this section we give a precise definition of the $\star$-algebra $\mathfrak{A}$ following (modulo a small improvement) the definition proposed by Sahlmann [1] after whom we name the algebra. Sahlmann's results of [1] will provide a useful characterization of representations of $\mathfrak{A}$. However, before we conclude that, we need to show that every representation of the algebra $\mathrm{Cyl}^{\infty}$ admits a decomposition into the cyclic representations as every representation of the full commutative $C^{\star}$-algebra Cyl. Next, we outline Sahlmann's characterization of representations of $\mathfrak{A}$. 


\subsection{Definitions}

Consider the subalgebra $\mathfrak{A}$ of the algebra of the linear operators $\mathrm{Cyl}^{\infty} \rightarrow$ $\mathrm{Cyl}^{\infty}$ generated by the Ashtekar-Corichi-Zapata algebra. Introduce the $\star-$ operator in $\mathfrak{A}$ by specifying its action on the generating set:

$$
\Psi^{\star}:=\bar{\Psi}, \quad X_{S, f}^{\star}=-X_{S, f},
$$

where $\Psi \in \mathrm{Cyl}^{\infty}$ and $X_{S, f}$ is the derivative operator defined in (2.7). The $\star$ operation extends consistently and uniquely to an involutive antiisomorphism of the entire algebra $\mathfrak{A}$.

Definition 3.1 The Sahlmann holonomy-flux $\star$ algebra is the algebra $(\mathfrak{A}, \star)$. The quantization map ${ }^{\wedge}: \mathfrak{A}_{\mathrm{ACZ}} \rightarrow \mathfrak{A}$ is defined on the generators of the Ashtekar-Corichi-Zapata algebra as follows:

$$
\hat{\Psi}:=\Psi, \quad \hat{X}_{S, f}:=-i X_{S, f},
$$

This definition concludes an important part of the quantization. The classical elementary variables are assigned to appropriate quantum elementary variables:

$$
\left\{\begin{array}{l}
\Psi \mapsto \hat{\Psi} \\
E(S, f) \mapsto \hat{X}_{S, f}
\end{array} .\right.
$$

Throughout this paper, all the elements of the Sahlmann holonomy-flux $\star-$

algebra will be denoted by hatted letters $\hat{a}, \hat{b}, \ldots$, in the way consistent with (3.1).

The last step of the kinematical loop quantization is construction of a $*$-representation of the holonomy-flux $\star$-algebra. Because the Sahlmann algebra is not equipped with any norm we are not allowed to expect that one can represent the algebra by means of bounded operators on a Hilbert space. This leads to the following definition of a $*$-representation of $\mathfrak{A}$ :

Definition 3.2 Let $L(\mathcal{H})$ be a space of linear operators on a Hilbert space $\mathcal{H}$. We say that a map $\pi: \mathfrak{A} \rightarrow L(\mathcal{H})$ is a $*$-representation of $\mathfrak{A}$ on the Hilbert space $\mathcal{H}$ if:

1. there exists a dense subspace $\mathcal{D}$ of $\mathcal{H}$ such that

$$
\mathcal{D} \subset \bigcap_{\hat{a} \in \mathfrak{A}}\left[D(\pi(\hat{a})) \cap D\left(\pi^{*}(\hat{a})\right)\right],
$$

where $D(\pi(\hat{a}))$ denotes the domain of the operator $\pi(\hat{a})$; 
2. for every $\hat{a}, \hat{b} \in \mathfrak{A}$ and $\lambda \in \mathbb{C}$ the following conditions are satisfied on $\mathcal{D}$ :

$$
\begin{aligned}
\pi(\hat{a}+\hat{b}) & =\pi(\hat{a})+\pi(\hat{b}), & & \pi(\lambda \hat{a})=\lambda \pi(\hat{a}), \\
\pi(\hat{a} \hat{b}) & =\pi(\hat{a}) \pi(\hat{b}), & & \pi\left(\hat{a}^{\star}\right)=\pi^{*}(\hat{a}) .
\end{aligned}
$$

3. If given $\hat{a} \in \mathfrak{A}$ there exists a dense linear subspace $D \subset D(\pi(\hat{a}))$ such that $\left.\pi(\hat{a})\right|_{D}$ is closable, then $\pi(\hat{a})$ is equal to the closure of $\left.\pi(\hat{a})\right|_{D}$.

We will say that $\pi$ is nondegenerate iff $\pi(\hat{a}) v=0$ for every $\hat{a} \in \mathfrak{A}$ implies $v=0$.

These conditions mean in particular, that $\pi(\hat{a}) \mathcal{D} \subset \mathcal{D}$ and that every element $\hat{a}=\hat{a}^{\star}$ is represented by the symmetric operator $\pi(\hat{a})$. $\mathfrak{A}$.

In the sequel we will consider only nondegenerate $*$-representations of

There is known a natural (nondegenerate) *-representation $\pi_{\mathrm{AL}}$ defined by the natural measure $\mu_{\mathrm{AL}}$ introduced [7, 8] in the Gel'fand-Neimark spectrum $\overline{\mathcal{A}}$ of $\mathrm{Cyl}$ (see also Section 4.2 ). The domain of the representation is $\mathrm{Cyl}^{\infty} \subset L^{2}\left(\overline{\mathcal{A}}, \mu_{\mathrm{AL}}\right)$ and $\pi_{\mathrm{AL}}$ is just the identity map. The representation has a large group of symmetries containing all the analytic diffeomorphisms of $\Sigma$ (we devote Section 4 to the issue of the diffemorphism invariance/covariance). Remarkably, the natural measure $\mu_{\mathrm{AL}}$ is the only measure with respect to which the identity map is a $\star$-algebra representation. We prove this uniqueness in Section 6.2

\subsection{Representations of $\mathrm{Cyl}{ }^{\infty}$}

Recently, Sahlmann started a new program of systematic study of the representation theory of the holonomy-flux $\star$-algebra $\mathfrak{A}$. To merge Sahlmann's conclusions on the representation theory of the $\star$-algebra $\mathfrak{A}$ we need one connecting lemma (this is a consequence of the difference in definitions between the current algebra $\mathfrak{A}$ and that of [1]).

Notice first that if $\pi$ is a nondegenerate $*$-representation of $\mathfrak{A}$ and $v \in \mathcal{D}$ then for every $\hat{a}$

$$
\pi(\hat{a})(v-\pi(\hat{1}) v)=0
$$

( $\hat{1}$ is the unit of the Sahlmann algebra defined by the function $\mathrm{Cyl}^{\infty} \ni$ $\Psi \equiv 1)$, hence by virtue of the nondegeneracy assumption $\pi(\hat{1})=$ id on $\mathcal{D}$ and consequently on the whole $\mathcal{H}$. This means in particular that the *-representation $\varrho$ of $\mathrm{Cyl}^{\infty}$ defined as $\varrho:=\left.\pi\right|_{\mathrm{Cyl}^{\infty}}$ is nondegenerate. 
Lemma 3.1 Suppose $\varrho$ is a nondegenerate *-representation of $\mathrm{Cyl}^{\infty}$ on $\mathcal{H}$ in the sense of Definition 3.2. Then $\varrho$ maps $\mathrm{Cyl}^{\infty}$ into the $C^{\star}$-algebra $B(\mathcal{H})$ of the bounded operators on $\mathcal{H}$ and admits a unique extension to a $C^{\star}$-algebra homomorphism of $\mathrm{Cyl}$ into $B(\mathcal{H})$.

Proof. We will show that for every $\Psi \in \mathrm{Cyl}^{\infty}$ and every $v \in \mathcal{D}$ the following inequality is true,

$$
\|\varrho(\Psi) v\|_{\mathcal{H}} \leq\|\Psi\|_{\text {sup }}\|v\|_{\mathcal{H}}
$$

where our notation distinguishes the Hilbert space norm in $\mathcal{H}$ from the supnorm in the $C^{\star}$-algebra Cyl. Given the inequality, it is obvious that: $(i)$ for every $\Psi \in \mathrm{Cyl}^{\infty}$ the operator $\varrho(\Psi)$ admits a unique extension to a bounded operator defined in $\mathcal{H},($ ii $) \varrho$ admits a unique extension to a linear and continuous map $\varrho: \mathrm{Cyl} \rightarrow B(\mathcal{H})$, and $(i i i)$ it is straightforward to check that the resulting extension of $\varrho$ onto $\mathrm{Cyl}$ is a $C^{\star}$-algebra homomorphism.

To show (3.3) fix an arbitrary number $q>1$ and notice that for every $\Psi \in \mathrm{Cyl}^{\infty}$ there exists an element $\Phi \in \mathrm{Cyl}^{\infty}$ such that $^{2}$

$$
\Phi \Phi^{*}+\Psi \Psi^{*}=q^{2}\|\Psi\|_{\text {sup }}^{2}
$$

Indeed, according to the definition of $\mathrm{Cyl}^{\infty}$, there is a graph $\gamma=\left\{e_{1}, \ldots, e_{N}\right\}$ and a smooth function $\psi: G^{N} \rightarrow \mathbb{C}$, such that $\Psi(A)=\psi\left(A\left(e_{1}\right), \ldots, A\left(e_{N}\right)\right)$ for every $A \in \mathcal{A}$. Define a new function $\Phi(A)=\phi\left(A\left(e_{1}\right), \ldots, A\left(e_{N}\right)\right)$ where

$$
\phi:=\sqrt{q^{2}\|\psi\|_{\mathrm{sup}}^{2}-|\psi|^{2}} .
$$

Notice that the function under the square root is non-negative and never vanishes, therefore the square is uniquely defined and the smoothness of $\psi$ implies the smoothness $\phi$.

Using (3.4) and the equality $\varrho(1)=$ id we find that for arbitrary $v \in \mathcal{D}$ :

$$
\|\varrho(\Psi) v\|_{\mathcal{H}}^{2}=q^{2}\|\Psi\|_{\text {sup }}^{2}\|v\|_{\mathcal{H}}^{2}-\|\varrho(\Phi) v\|_{\mathcal{H}}^{2} \leq q^{2}\|\Psi\|_{\text {sup }}^{2}\|v\|_{\mathcal{H}}^{2} .
$$

Given $\Psi$ and $v$, the inequality holds for every $q>1$, therefore it is true also for $q=1$. This completes the proof of the lemma.

\subsection{Sahlmann's characterization of representations of $\mathfrak{A}$}

Starting from this point we can directly apply Sahlmann's arguments [1] leading to a useful characterization of a quite broad class of representations of the holonomy-flux $\star$-algebra $\mathfrak{A}$. Suppose $\pi$ is a nondegenerate *representation of $\mathfrak{A}$ in a Hilbert space $\mathcal{H}$. Consider first the restriction of

\footnotetext{
${ }^{2}$ This fact was pointed out to us by Professor S. L. Woronowicz.
} 
$\pi$ to the subalgebra $\mathrm{Cyl}^{\infty}$. According to Lemma 3.1 $\left.\pi\right|_{\mathrm{Cyl}}{ }^{\infty}$ extends to a representation of the $C^{\star}$-algebra $\mathrm{Cyl}$ in $\mathcal{H}$, therefore it admits the following decomposition described by

\section{Characterization 3.1 (Sahlmann)}

1. Representation $\left.\pi\right|_{\mathrm{Cy} 1^{\infty}}$ is the direct sum of cyclic representations $\left\{\pi_{\nu}\right\}$ :

$$
\mathcal{H}=\bigoplus_{\nu \in \mathcal{N}} \mathcal{H}_{\nu},\left.\quad \pi\right|_{\mathrm{Cyl}} ^{\infty}=\bigoplus_{\nu \in \mathcal{N}} \pi_{\nu},
$$

where $\left\{\mathcal{H}_{\nu}\right\}$ are carrier spaces of representations $\left\{\pi_{\nu}\right\}$ respectively, $\nu$ ranges some label set $\mathcal{N}$ and the sum is orthogonal;

2. For each $\nu$ :

$$
\mathcal{H}_{\nu}=L^{2}\left(\overline{\mathcal{A}}, \mu_{\nu}\right)
$$

where $\overline{\mathcal{A}}$ is a Gel'fand-Neimark spectrum ${ }^{3}$ of $\mathrm{Cyl}$, and $\mu_{\nu}$ is a regular, Borel measure on $\overline{\mathcal{A}}$ defined by a positive, linear functional $\mathrm{Cyl} \rightarrow \mathbb{C}$.

3. Let $\Psi \in L^{2}\left(\overline{\mathcal{A}}, \mu_{\nu}\right)$ and $\Phi \in \mathrm{Cyl}^{\infty}$. Then:

$$
\pi(\hat{\Phi}) \Psi=\Phi \Psi .
$$

Given $\Psi \in \mathcal{H}$ we will denote by $\Psi_{\nu}$ the orthogonal projection of $\Psi$ onto $\mathcal{H}_{\nu}$, and write $\Psi=\left(\Psi_{\nu}\right)$.

Turn now to the whole representation $\pi$. Following [1] we define:

$$
\begin{aligned}
& \mathcal{C}^{\infty}:=\left\{\left(\Psi_{\nu}\right) \in \mathcal{H} \mid \Psi_{\nu}=0 \text { for all but finitely many } \nu\right. \text { 's } \\
& \text { and } \left.\Psi_{\nu} \in \mathrm{Cyl}^{\infty} \text { for every } \nu\right\} .
\end{aligned}
$$

It turns out that the assumption $\mathcal{D}=\mathcal{C}^{\infty}$, where $\mathcal{D}$ is introduced by Definition 3.2 gives the following useful characterization:

Characterization 3.2 (Sahlmann) Suppose $\pi$ is a nondegenerate $*$-representation of $\mathfrak{A}$ in a Hilbert space $\mathcal{H}$, and $\pi$ satisfies the assumption $\mathcal{D}=$ $\mathcal{C}^{\infty}$. Then $\mathcal{H}$ and the restriction of $\pi$ to $\mathrm{Cyl}^{\infty}$ satisfy the decomposition given by Characterization [3.1. Moreover, for every $\hat{X}_{S, f}, \pi$ defines a family of elements of $\mathcal{H}$ labelled by elements of the labelling set $\mathcal{N}$ (the same as in (3.73.8)

$$
\mathcal{N} \ni \iota \mapsto F_{S, f} \iota \in \mathcal{H},
$$

such that the following conditions are satisfied (given $F_{S, f}{ }^{\iota}$ we will subsequently denote by $F_{S, f^{\iota}}{ }_{\nu}$ the $\mathcal{H}_{\nu}$ component of $\left.F_{S, f^{\iota}}\right)$ :

\footnotetext{
${ }^{3}$ For more details about the space $\overline{\mathcal{A}}$ see Subsection 4.2
} 
1. for every $\Psi=\left(\Psi_{\nu}\right) \in \mathcal{C}^{\infty}$ :

$$
\pi\left(\hat{X}_{S, f}\right) \Psi=\hat{\mathbf{X}}_{S, f} \Psi+\hat{F}_{S, f} \Psi
$$

where $\hat{\mathbf{X}}_{S, f} \Psi:=\left(\hat{X}_{S, f} \Psi_{\nu}\right)$ and:

$$
\hat{F}_{S, f} \Psi=\hat{F}_{S, f}\left(\Psi_{\nu}\right):=\left(\sum_{\iota} \Psi_{\iota} F_{S, f_{\nu}^{\iota}}\right)
$$

where $F_{S, f^{\iota}}{ }_{\nu} \in L^{2}\left(\overline{\mathcal{A}}, \mu_{\nu}\right)$;

2. for every $\Phi, \Phi^{\prime} \in \mathrm{Cyl}^{\infty} \subset L^{2}\left(\overline{\mathcal{A}}, \mu_{\nu}\right)$ :

$$
\left\langle\hat{X}_{S, f} \Phi \mid \Phi^{\prime}\right\rangle_{\nu}-\left\langle\Phi \mid \hat{X}_{S, f} \Phi^{\prime}\right\rangle_{\nu}=\left\langle\Phi \mid\left(F_{S, f^{\nu}}{ }^{\nu}-\bar{F}_{S, f^{\nu}}{ }^{\nu}\right) \Phi^{\prime}\right\rangle_{\nu},
$$

where $\langle\cdot \mid \cdot\rangle_{\nu}$ is the scalar product on $L^{2}\left(\overline{\mathcal{A}}, \mu_{\nu}\right)$ (no summation with respect to the index $\nu \in \mathcal{N}$ in $\left.F_{S, f}{ }_{\nu}{ }^{\prime}\right)$;

3. for every $S=S_{1} \cup S_{2}$ such that $S_{i}(i=1,2)$ are disjoint:

$$
F_{S, f_{\nu}{ }^{\iota}}=F_{S_{1}, f_{1}{ }^{\iota}{ }_{\nu}}+F_{S_{2}, f_{2}{ }^{\iota}}
$$

where $f_{i}:=\left.f\right|_{S_{i}}$.

Sahlmann's characterization of [1] contains a more exhaustive discussion; we only outlined those elements which will be relevant in the current paper.

From the point of view of the loop quantization program, fixing a specific representation $\pi$ of the Sahlmann holonomy-flux $\star$-algebra completes the (kinematical) quantization. The composition of $\pi$ with the mapping ^ (3.2) becomes a quantum representation of the classical elementary variables as quantum operators in $\mathcal{H}$ :

$$
\left\{\begin{array}{l}
\Phi \mapsto \pi(\hat{\Phi}) \\
E_{S, f} \mapsto \pi\left(\hat{X}_{S, f}\right)
\end{array} .\right.
$$

In particular, $\pi\left(\hat{X}_{S, f}\right)$ is the quantum flux operator.

\section{A diffeomorphism symmetry and statement of the problem}

\subsection{Statement of the problem}

The smooth diffeomorphisms of $\Sigma$ act naturally in the space of connections $\mathcal{A}$. The action induces an action of the analytic diffeomorphisms in the 
$C^{\star}$-algebra Cyl and finally in the Sahlmann holonomy-flux $\star$-algebra $\mathfrak{A}$.( In this paper we consider the analytic diffeomorphisms only and henceforth we will drop the word 'analytic'.) However, imposing the diffeomorphism covariance on a representation $\pi$ of the Sahlmann algebra is a priori ambiguous. Therefore, we formulate now a precise definition of the diffeomorphism covariance considered in this paper. Given a diffeomorphism $\varphi: \Sigma \rightarrow \Sigma$, denote by $\tilde{\varphi}$ the induced action $\tilde{\varphi}: \mathrm{Cyl}^{\infty} \rightarrow \mathrm{Cyl}^{\infty}$. Consider first a simple case when $\pi_{\mid \mathrm{Cy}} \infty$ is a cyclic representation, that is when the decomposition (3.73.8) consists of a single term only. The representation $\pi$ will be called diffeomorphism covariant if there is a cyclic element $v \in \mathcal{H}$ such that the state it defines in $\mathfrak{A}$ is diffeomorphism invariant:

$$
\left\langle v \mid \pi\left(\tilde{\varphi} \hat{a} \tilde{\varphi}^{-1}\right) v\right\rangle=\langle v \mid \pi(\hat{a}) v\rangle
$$

for every $\hat{a} \in \mathfrak{A}$ and for every diffeomorphism $\varphi$. A general definition we formulate is:

Definition 4.1 Suppose $\pi$ is a nondegenerate *-representation of the Sahlmann $(\mathfrak{A}, \star)$ in a Hilbert space $\mathcal{H}$ described by Characterization [3.1. The representation $\pi$ is called covariant with respect to the diffeomorphisms of $\Sigma$ if each of the components $\mathcal{H}_{\nu}$ in (3.73.8) contains a cyclic ${ }^{4}$ vector $v_{\nu}$ such that every finite linear combination $v$ of the vectors $v_{\nu}, \nu \in \mathcal{N}$ defines a diffeomorphism invariant state in $\mathfrak{A}$; that is, when:

$$
\left\langle v \mid \pi\left(\tilde{\varphi} \hat{a} \tilde{\varphi}^{-1}\right) v\right\rangle=\langle v \mid \pi(\hat{a}) v\rangle
$$

for every $a \in \mathfrak{A}$, and every diffeomorphism $\varphi$.

We will see in Subsection 4.4 that every diffeomorphism maps a diffeomorphism covariant representation into a unitarily equivalent one. In this sense the covariance implies the invariance.

Importantly, the representation used in the loop quantization programwhich is given by $\mathcal{H}=L^{2}\left(\overline{\mathcal{A}}, \mu_{\mathrm{AL}}\right)$ with the natural measure $\mu_{\mathrm{AL}}$ and all the correction functions $F_{S, f^{\iota}{ }_{\nu}}=0$-is diffeomorphism covariant in the sense of Definition 4.1]

A working version of the definition of the covariance was assumed by Sahlmann in [2]. He shows that in the $G=U(1)$ case a diffeomorhism covariant cyclic representation $\pi$ necessarily corresponds to the natural measure $\mu_{\mathrm{AL}}$ mentioned in the Section 3.1 (see also the next subsection).

\footnotetext{
${ }^{4}$ We mean here, that $v_{\nu}$ is cyclic with respect to the corresponding representation $\pi_{\nu}$ defined by (3.7).
} 
The goal of this paper is to study the diffeomorphism covariant representations $\pi$ in the general case of Characterization 3.2 and to derive the resulting restrictions on the measures $\mu_{\nu}$ in the decomposition (3.7/3.8) and

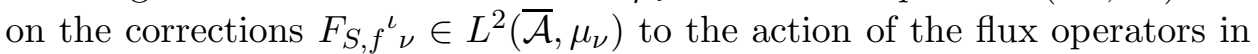
the general compact and connected gauge group $G$ case.

In the remaining part of this section we outline the facts concerning the measures on $\overline{\mathcal{A}}$ and the space $\overline{\mathcal{A}}$ itself which will be used in the next section. In particular we recall the definition of the natural measure $\mu_{\mathrm{AL}}$. Finally, we formulate the diffeomorpfism invariance/covariance condition in terms of Characterization 3.2

\subsection{The measures on $\overline{\mathcal{A}}$}

The $C^{\star}$-algebra $\mathrm{Cyl}$ is isomorphic with the algebra of the continuous functions on the compact Hausdorff space $\overline{\mathcal{A}}$ of the generalized connections. The space $\overline{\mathcal{A}}$ can be naturally identified with the space of maps $\bar{A}: \mathbf{E} \rightarrow G$, where $\mathbf{E}$ is the space of the edges in $\Sigma$, such that [4:

$$
\begin{aligned}
\bar{A}\left(e^{-1}\right) & =[\bar{A}(e)]^{-1} \\
\bar{A}\left(e_{2} \circ e_{1}\right) & =\bar{A}\left(e_{2}\right) \bar{A}\left(e_{1}\right)
\end{aligned}
$$

for every triple of edges $e_{1}, e_{2}, e$. Obviously, an example is the holonomy map (2.3). Given $\bar{A}$, the corresponding element of $\overline{\mathcal{A}}$ is the homomorphism $\mathrm{Cyl}^{0} \ni \Psi \mapsto \psi\left(\bar{A}\left(e_{1}\right), \ldots, \bar{A}\left(e_{N}\right)\right) \in \mathbb{C}$ where $\Psi$ is written as in (2.4).

A convenient tool for description measures on $\overline{\mathcal{A}}$ is a family of quotient spaces labelled by the graphs in $\Sigma$, obtained from $\overline{\mathcal{A}}$ and equipped with a family of some projective maps. A graph $\gamma=\left\{e_{1}, \ldots, e_{N}\right\}$ defines an equivalence relation on $\overline{\mathcal{A}}$ :

$$
\bar{A}_{1} \sim_{\gamma} \bar{A}_{2} \Longleftrightarrow \bar{A}_{1}\left(e_{I}\right)=\bar{A}_{2}\left(e_{I}\right)
$$

for every $I=1, \ldots, N$. This relation defines the quotient space $\overline{\mathcal{A}}_{\gamma}$,

$$
\overline{\mathcal{A}}_{\gamma}:=\overline{\mathcal{A}} / \sim_{\gamma}
$$

and the projection $p_{\gamma}: \overline{\mathcal{A}} \rightarrow \overline{\mathcal{A}}_{\gamma}$. The map $\overline{\mathcal{A}} \ni \bar{A} \mapsto\left(\bar{A}\left(e_{1}\right), \ldots, \bar{A}\left(e_{N}\right)\right)$ induces a bijective map

$$
\overline{\mathcal{A}}_{\gamma} \leftrightarrow G^{N}
$$

which equips the space $\overline{\mathcal{A}}_{\gamma}$ with the geometry of $G^{N}$. Moreover, for every equivalence class $[\bar{A}]_{\gamma} \in \overline{\mathcal{A}}_{\gamma}$ there exists $A \in \mathcal{A}$ such that $A \in[\bar{A}]_{\gamma}$ [12, 13]. 
Thus every function $\psi: G^{N} \rightarrow \mathbb{C}$ defining cylindrical function $\Psi$ compatible with a graph $\gamma$ can be regarded as a function on $\overline{\mathcal{A}}_{\gamma}$ (see Definition 2.4).

Finally, we recall the projective family structure of the family of the spaces $\left\{\overline{\mathcal{A}}_{\gamma}\right\}$ labelled by all the graphs $\gamma$ in $\Sigma$. Notice first that graphs in $\Sigma$ form a partially ordered, directed set with relation $\geq$ defined as follows: $\gamma^{\prime} \geq \gamma$ if every edge of $\gamma$ can be expressed as a composition of edges of $\gamma^{\prime}$ (and their inverses) and each vertex of $\gamma$ is a vertex of $\gamma^{\prime}$. Now, if $\gamma^{\prime} \geq \gamma$ then there exists a projection $p_{\gamma \gamma^{\prime}}: \overline{\mathcal{A}}_{\gamma^{\prime}} \rightarrow \overline{\mathcal{A}}_{\gamma}$ such that:

$$
p_{\gamma}=p_{\gamma \gamma^{\prime}} \circ p_{\gamma^{\prime}}
$$

An important application of $\left\{\overline{\mathcal{A}}_{\gamma}, p_{\gamma}, p_{\gamma \gamma^{\prime}}\right\}$ is the construction of measures on $\overline{\mathcal{A}}$ from projective families of measures defined on the spaces $\overline{\mathcal{A}}_{\gamma}$. Every measure $\mu$ defined on $\overline{\mathcal{A}}$ defines a family of measures $\left\{\mu_{\gamma}:=p_{\gamma^{*}} \mu\right\}$ on $\left\{\overline{\mathcal{A}}_{\gamma}\right\}$ respectively:

$$
\int_{\overline{\mathcal{A}}_{\gamma}} \psi d \mu_{\gamma}=\int_{\overline{\mathcal{A}}} \Psi d \mu
$$

for every $\Psi \in \mathrm{Cyl}^{0}$ compatible with a graph $\gamma$ (in the latter equation $\Psi$ is related to $\psi$ as in Definition 2.4). If $\Psi$ is compatible with two graphs $\gamma, \gamma^{\prime}$, then:

$$
\int_{\overline{\mathcal{A}}_{\gamma}} \psi d \mu_{\gamma}=\int_{\overline{\mathcal{A}}_{\gamma^{\prime}}} \psi^{\prime} d \mu_{\gamma^{\prime}}
$$

Thus in the case of $\gamma^{\prime} \geq \gamma$ we have:

$$
\left(p_{\gamma \gamma^{\prime}}\right)_{*} \mu_{\gamma^{\prime}}=\mu_{\gamma}
$$

Conversely, if for every graph $\gamma$ we endow the space $\overline{\mathcal{A}}_{\gamma}$ with a measure $\mu_{\gamma}$, such that the resulting family of measures is consistent in the sense of Equation (4.4) for every pair of graphs $\gamma^{\prime} \geq \gamma$, then the following integration functional:

$$
\mathrm{Cyl}^{0} \ni \Psi \mapsto \int_{\overline{\mathcal{A}}_{\gamma}} \psi d \mu_{\gamma} \in \mathbb{C}
$$

is positive definite hence defines a regular, Borel measure on $\overline{\mathcal{A}}[12$.

The natural measure $\mu_{\mathrm{AL}}$ is defined by choosing each $\mu_{\gamma}$ to be the measure given by the probability Haar measure on $G^{N}$ and by the bijection (4.11). 


\subsection{The action of the diffeomorphisms in the Sahlmann al- gebra}

Let $\varphi$ be a diffeomorphism on $\Sigma$. It acts in a natural way on pair of fields $(A, \tilde{E})$ :

$$
A \mapsto \varphi^{*} A, \quad \tilde{E} \mapsto \varphi_{*}^{-1} \tilde{E} .
$$

This induces an action $\tilde{\varphi}$ of $\varphi$ on the cylindrical functions (2.4) and the fluxes:

$$
\begin{gathered}
(\tilde{\varphi} \Phi)(A):=\phi\left(A\left(\varphi\left(e_{1}\right)\right), \ldots, A\left(\varphi\left(e_{N}\right)\right)\right) \\
\tilde{\varphi} E(S, f):=E(\tilde{S}, \tilde{f}), \\
\tilde{S}=\varphi(S), \quad \tilde{f}=\left(\left.\varphi^{-1}\right|_{S}\right)^{*} f .
\end{gathered}
$$

The derivations $X_{S, f}$ are defined in a diffeomorphism covariant way; that is, for every $\Phi \in \mathrm{Cyl}^{\infty}$, flux $E(S, f)$ and diffeomorphism $\varphi$ we have

$$
\tilde{\varphi}\left(X_{S, f} \Phi\right)=X_{\tilde{S}, \tilde{f}} \tilde{\varphi} \Phi .
$$

Notice that by using the characterization of the space $\overline{\mathcal{A}}$ as the space of maps $\bar{A}: \mathbf{E} \rightarrow G$, the diffemorphisms of $\Sigma$ act naturally in $\overline{\mathcal{A}}$ via $\left(\varphi^{*} \bar{A}\right)(e):=$ $\bar{A}(\varphi(e))$. This action is consistent with the extension of the cylindrical functions to functions defined on $\overline{\mathcal{A}}$ and with the action (4.7).

The Sahlmann algebra $\mathfrak{A}$ is a subalgebra of linear operators acting in $\mathrm{Cyl}^{\infty}$, thus the action $\tilde{\varphi}: \mathrm{Cyl}^{\infty} \rightarrow \mathrm{Cyl}^{\infty}$ induces the following action of $\varphi$ on $\mathfrak{A}$ :

$$
\mathfrak{A} \ni \hat{a} \mapsto \tilde{\varphi} \hat{a} \tilde{\varphi}^{-1} \in \mathfrak{A} .
$$

The action is a clearly a $\star$-algebra isomorphism. In particular, we have the following action for the generators. For every $\hat{\Phi}$, where $\Phi \in \mathrm{Cyl}^{\infty}$, and for every derivation operator $\hat{X}_{S, f}$, the action reads

$$
\tilde{\varphi} \hat{\Phi} \tilde{\varphi}^{-1}=\widehat{\tilde{\varphi} \Phi}, \quad \tilde{\varphi} \hat{X}_{S, f} \tilde{\varphi}^{-1}=\hat{X}_{\tilde{S}, \tilde{f}}
$$

\subsection{The diffeomorphism covariance conditions in terms of Characterization 3.1}

Consider now a representation $\pi$ of the Sahlmann $\star$-algebra $\mathfrak{A}$ in a Hilbert space $\mathcal{H} \bigoplus_{\nu \in \mathcal{N}} \mathcal{H}_{\nu}$ given by Characterization 3.1 and assume it is covariant with respect to the diffeomorphisms of $\Sigma$ in the sense of Definition 4.1 The family of the vectors $v_{\nu}, \nu \in \mathcal{N}$ provided by the definition can be used to induce a unitary action $U_{\varphi}$ of a diffeomorphism $\varphi$ of $\Sigma$ in $\mathcal{H}$. Then, 
the conditions of Definition 4.1 turn into appropriate invariance/covariance conditions with respect that action. We spell them out in this subsection.

Notice first that we have the following identification (see (3.8)):

$$
L^{2}\left(\overline{\mathcal{A}}, \mu_{\nu}\right) \supset \operatorname{Cyl} \ni \Phi \leftrightarrow \pi(\hat{\Phi}) v_{\nu} \in \mathcal{H}_{\nu}
$$

Using this it is easy to see that Definition 4.1 implies the equality $\mathcal{D}=\mathcal{C}^{\infty}$. Consequently Characterization 3.2 is true as well.

The induced action $u_{\varphi}$ of a diffeomorphism $\varphi$ in each $\mathcal{H}_{\nu}$ is defined by:

$$
u_{\varphi} \pi(\hat{\Phi}) v_{\nu}:=\pi\left(\tilde{\varphi} \hat{\Phi} \tilde{\varphi}^{-1}\right) v_{\nu}=\pi(\widehat{\tilde{\varphi} \Phi}) v_{\nu},
$$

where $\Phi \in \mathrm{Cyl}$ and the existence of a unique extension of $\pi_{\mid \mathrm{Cyl}}{ }^{\infty}$ to the entire $\mathrm{Cyl}^{\star}$ algebra completion $\mathrm{Cyl}$ is used. The corresponding action of $\varphi$ in $\mathcal{H}$ will be denoted by $U_{\varphi}$.

The first consequence of the diffeomorphism covariance of the representation $\pi$ (Definition 4.1) is that, $\varphi \mapsto U_{\varphi}$ is a unitary representation of the diffeomorphism group in $\mathcal{H}$, and $\varphi \mapsto u_{\varphi}$ is a unitary representation of the diffeomorphism group in $\mathcal{H}_{\nu}$ for each cyclic component $\mathcal{H}_{\nu}$.

It is easy to see that the second consequence of the diffeomorphism covariance is that $\pi$ is covariant with the actions of the diffeomorphisms in the algebra $\mathfrak{A}$ and in the Hilbert space $\mathcal{H}$. That is, for every diffeomorphism $\varphi$ and every $\hat{a} \in \mathfrak{A}$ the equality

$$
\pi\left(\tilde{\varphi} \hat{a} \tilde{\varphi}^{-1}\right)=U_{\varphi} \pi(\hat{a}) U_{\varphi}^{-1}
$$

holds on $\mathcal{C}^{\infty}$.

Every diffeomorphism invariant state $v_{\nu}$ defines a diffeomorphism invariant measure on $\overline{\mathcal{A}}$ :

$$
\int_{\overline{\mathcal{A}}} \Psi d \mu_{\nu}:=\left\langle v_{\nu} \mid \pi(\hat{\Psi}) v_{\nu}\right\rangle
$$

Indeed, in terms of the isomorphism (4.10) the action of $u_{\varphi}$ in $\mathcal{H}_{\nu}$ coincides with the action of $\tilde{\varphi}$ in $L^{2}\left(\overline{\mathcal{A}}, \mu_{\nu}\right)$. The unitarity of $u_{\varphi}$ is equivalent to the invariance of the measures $\mu_{\nu}, \nu \in \mathcal{N}$ with respect to the action $\tilde{\varphi}$ of each diffeomorphism $\varphi$.

Now we can state that:

Corollary 1 If $\pi$ is a nondegenerate, diffeomorphism covariant *-representation of $\mathfrak{A}$, then $\pi$ satisfies requirements of Characterizations [3.1 and 3.2. Moreover, the three conditions: $(i)$ the unitarity of the operators $U_{\varphi}$, (ii) $\mathcal{D}=\mathcal{C}^{\infty}$ and (iii) the diffeomorphism covariance condition (4.11) are equivalent to Definition 4.1. 
In particular, taking into account (4.9) we have for every operator $\hat{X}_{S, f}$,

$$
\pi\left(\hat{X}_{\tilde{S}, \tilde{f}}\right)=U_{\varphi} \pi\left(\hat{X}_{S, f}\right) U_{\varphi}^{-1}
$$

(the latter and the next two equations hold on $\mathcal{C}^{\infty}$ ). On the other hand (4.8) implies:

$$
\hat{\mathbf{X}}_{\tilde{S}, \tilde{f}}=U_{\varphi} \hat{\mathbf{X}}_{S, f} U_{\varphi}^{-1} .
$$

Since $\pi\left(\hat{X}_{S, f}\right)=\hat{\mathbf{X}}_{S, f}+\hat{F}_{S, f}$ according to Characterization 3.2. the diffeomorphism covariance of $\pi$ implies the following covariance of $\hat{F}_{S, f}$ :

$$
\hat{F}_{\tilde{S}, \tilde{f}}=U_{\varphi} \hat{F}_{S, f} U_{\varphi}^{-1}
$$

where $\tilde{S}=\varphi(S), \tilde{f}=\left(\left.\varphi^{-1}\right|_{S}\right)^{*} f$.

\section{Main theorem}

Let us formulate the theorem which is the main result of this paper.

We consider the Sahlmann $\star$-algebra $(\mathfrak{A}, \star)$ (Definition 3.1) corresponding to the space of the $G$-connections defined on the trivial bundle $\Sigma \times G$ where $G$ is a compact, connected Lie group. The natural measure defined on the space $\overline{\mathcal{A}}$ of the generalized connections was recalled in Section 4.2

Theorem 5.1 Suppose $\pi$ is a nondegenerate *-representation of the Sahlmann algebra $\mathfrak{A}$. Suppose also, that $\pi$ is covariant with respect to the group of the (analytic) diffeomorphisms of $\Sigma$ in the meaning of Definition 4.1. and $\Sigma=\mathbb{R}^{d}$. Then, for every $\nu \in \mathcal{N}$, the measure $\mu_{\nu}$ in Characterization 3.1 corresponding to the diffeomorphism invariant state $v_{\nu}$ of Definition 4.1 is the natural measure:

$$
\mu_{\nu}=\mu_{\mathrm{AL}}
$$

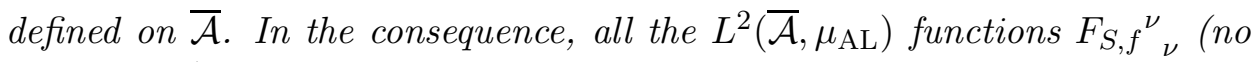
summation) used in Characterization 3.2 are real valued.

\section{Remarks}

1. The only reason for the (restrictive) assumption $\Sigma=\mathbb{R}^{d}$ is that in the proof below, we will need some special analytic diffeomorphisms on $\mathbb{R}^{d}$. Thus far we were able to construct the needed diffeomorphisms only in the $\Sigma=\mathbb{R}^{d}$ case. 
2. Taking into account Lemma 3.1 we can see that upon the requirement of nondegeneracy and the defintion of the diffeomorphism covariance, every representation $\pi$ of the Sahlmann $\star$-algebra satisfies the conclusions of the Theorem 5.1 .

\section{Proof of the main theorem}

Corollary 1 guarantees that every representation $\pi$ satisfying assumptions of the main theorem satisfies conditions of Characterizations 3.1 and 3.2 According to the latter one the quantum flux operator $\pi\left(\hat{X}_{S, f}\right)$ has the following form:

$$
\pi\left(\hat{X}_{S, f}\right)=\hat{\mathbf{X}}_{S, f}+\hat{F}_{S, f}
$$

for every submanifold $S$ and smearing function $f$.

Our proof consists of two steps. First, we will show that whenever $S$ is diffeomorphic to a $(d-1)$-dimensional (coordinate) cube $C$ and $f: S \rightarrow G^{\prime}$ is a constant function, then the imaginary part of $F_{C, f^{\nu} \nu}$ has to be equal zero for every $\nu$. Therefore, the corresponding operator $\hat{X}_{C, f}$ restricted to $\mathrm{Cyl}^{\infty} \subset L^{2}\left(\overline{\mathcal{A}}, \mu_{\nu}\right)$ is symmetric with respect to the scalar product on $L^{2}\left(\overline{\mathcal{A}}, \mu_{\nu}\right)$.

The symmetry of the operator $\hat{X}_{C, f}$ will be enough to conclude in the second step of the proof that, for every $\nu$, the measure $\mu_{\nu}$ is the natural measure $\mu_{\mathrm{AL}}$.

\subsection{Step 1: The imaginary part of $F_{C, f^{\nu}}{ }^{\nu}$}

\subsubsection{The functions $F_{S, f^{\iota} \nu}$}

Consider the functions $F_{S, f^{\iota} \nu}$ and their imaginary parts:

$$
I_{S, f^{\iota} \nu}:=\frac{1}{2}\left(F_{S, f^{\iota} \nu}-\bar{F}_{S, f^{\prime} \nu}\right) \in L^{2}\left(\overline{\mathcal{A}}, \mu_{\nu}\right) .
$$

The assumed diffeomorphism covariance of representation $\pi$ allows us to make use of the results derived in Subsection 4.4. In particular Equation (4.14) implies

$$
U_{\varphi} \hat{F}_{S, f} \Psi=\hat{F}_{\tilde{S}, \tilde{f}} U_{\varphi} \Psi
$$

for every diffeomorphism $\varphi$ and for every $\Psi \in \mathcal{C}^{\infty}$. The l.h.s. of the above 
equation can be transformed in the following way:

$$
\begin{aligned}
U_{\varphi} \hat{F}_{S, f} \Psi=U_{\varphi}\left(\sum_{\iota} F_{S, f^{\iota} \nu} \Psi_{\iota}\right)=\left(\sum_{\iota} u_{\varphi}\left(F_{S, f^{\iota} \nu} \Psi_{\iota}\right)\right)= & \\
& =\left(\sum_{\iota} u_{\varphi}\left(F_{S, f^{\iota} \nu}\right) u_{\varphi}\left(\Psi_{\iota}\right)\right),
\end{aligned}
$$

while the r.h.s. is:

$$
\hat{F}_{\tilde{S}, \tilde{f}} U_{\varphi} \Psi=\hat{F}_{\tilde{S}, \tilde{f}} U_{\varphi}\left(\Psi_{\nu}\right)=\hat{F}_{\tilde{S}, \tilde{f}}\left(u_{\varphi} \Psi_{\nu}\right)=\left(\sum_{\iota} F_{\tilde{S}, \tilde{f} \nu} u_{\varphi}\left(\Psi_{\iota}\right)\right) .
$$

A comparison of the two latter results shows that the elements $F_{S, f}$ of $\mathcal{H}$ are assigned to the submanifolds $S$ and the functions $f$ in a covariant way,

$$
u_{\varphi} F_{S, f^{\iota} \nu}=F_{\tilde{S}, \tilde{f}^{\iota} \nu} \text {, hence } u_{\varphi} I_{S, f^{\iota} \nu}=I_{\tilde{S}, \tilde{f} \nu}^{\iota} .
$$

where $\tilde{S}=\varphi(S)$ and $\tilde{f}=\left(\left.\varphi^{-1}\right|_{S}\right)^{*} f$.

The diffemorphism covariance of the representation $\pi$ also implies that for every $\nu$ the scalar product $\langle\cdot \mid \cdot\rangle_{\nu}$ on $L^{2}\left(\overline{\mathcal{A}}, \mu_{\nu}\right)$ is diffeomorphism invariant, thus

$$
\left\|I_{S, f^{\iota} \nu}\right\|_{\nu}=\left\|u_{\varphi} I_{S, f^{\iota} \nu}\right\|_{\nu}=\left\|I_{\tilde{S}, \tilde{f}^{\iota} \nu}\right\|_{\nu}
$$

Equation (3.13) allows us to conclude, that for $S=S_{1} \cup S_{2}$, where $S_{1}$ and $S_{2}$ are disjoint

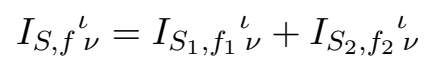

(here $f_{i}:=\left.f\right|_{S_{i}}$ and $\left.i=1,2\right)$.

Using the properties $(6.3,6.4)$ we will show in the next subsections, that for every $(d-1)$-dimensional cube $C$ in $\mathbb{R}^{d}$ and every constant function $f$

$$
I_{C, f^{\nu} \nu}=0 \text {. }
$$

\subsubsection{The functions $I_{C, f^{\nu} \nu}$ for cubes and constant functions $f$}

Consider in $\Sigma=\mathbb{R}^{d}$ an arbitrary coordinate system $\left(x^{j}\right),(j=1, \ldots, d)$ given by an affine transformation applied to the Cartesian coordinates. Let $C \subset \mathbb{R}^{d}$ be a cube given by the following inequalities:

$$
C:=\left\{y \in \mathbb{R}^{d} \mid\left\{\begin{array}{l}
x^{d}(y)=0 \\
-l<x^{1}(y) \leq l \\
-l<x^{i}(y)<l \quad \text { for } i=2,3, \ldots, d-1 .
\end{array} \quad ; \quad l>0\right\} .\right.
$$


Notice, that $C$ includes one of the sites, namely the one contained in the $x^{d}=0, x^{1}=l$ plane. Due to that subtlety, $C$ can be split by a suitable partition into two disjoint cubes $C_{1}, C_{2}$ diffeomorphic to $C$. The cubes can be defined as follows:

$$
\begin{aligned}
C_{1} & :=\left\{y \in C \mid x^{1}(y) \leq 0\right\} \\
C_{2} & :=\left\{y \in C \mid x^{1}(y)>0\right\} .
\end{aligned}
$$

Let us fix the index $\nu$ and denote $I_{C, f}=I_{C, f^{\nu}}{ }_{\nu}$ in order to simplify notation. Recall that $I_{C, f} \in L^{2}\left(\overline{\mathcal{A}}, \mu_{\nu}\right)$. We are going now to find some relation between $I_{C, f}$ and $I_{C_{i},\left.f\right|_{C_{i}}}(i=1,2)$ for a constant function $f$.

Assume then that $f$ is an arbitrary constant function on $C$. Because the cubes $C_{1}, C_{2}$ are diffeomorphic to $C$, then Equation (6.3) gives us:

$$
\left\|I_{C, f}\right\|_{\nu}^{2}=\left\|I_{C_{1}, f}\right\|_{\nu}^{2}=\left\|I_{C_{2}, f}\right\|_{\nu}^{2},
$$

$C=C_{1} \cup C_{2}$ and $C_{1} \cap C_{2}=\varnothing$, thus by virtue of Equation (6.4):

$$
I_{C, f}=I_{C_{1}, f}+I_{C_{2}, f} .
$$

Combining the two latter equations we obtain:

$$
\left\|I_{C, f}\right\|_{\nu}^{2}=\left\|I_{C_{1}, f}+I_{C_{2}, f}\right\|_{\nu}^{2}=2\left\|I_{C, f}\right\|_{\nu}^{2}+\left\langle I_{C_{1}, f} \mid I_{C_{2}, f}\right\rangle_{\nu}+\left\langle I_{C_{2}, f} \mid I_{C_{1}, f}\right\rangle_{\nu},
$$

that is:

$$
\left\|I_{C, f}\right\|_{\nu}^{2}=-2\left\langle I_{C_{1}, f} \mid I_{C_{2}, f}\right\rangle_{\nu} .
$$

Our goal now is to show, that the scalar product $\left\langle I_{C_{1}, f} \mid I_{C_{2}, f}\right\rangle_{\nu}$ is equal 0 . We will do it in two steps. First, we will express the function $I_{C_{1}, f}$ as a limit of a suitably chosen sequence of cylindrical functions belonging to $\mathrm{Cyl}^{\infty} \subset L^{2}\left(\overline{\mathcal{A}}, \mu_{\nu}\right)$. Then, Characterization 3.2 will allow us to conclude, that $\left\langle I_{C_{1}, f} \mid I_{C_{2}, f}\right\rangle_{\nu}=0$.

\subsubsection{The function $I_{C_{1}, f}$ as a limit of a sequence of cylindrical functions}

The function $I_{C_{1}, f}$ can be expressed as a limit,

$$
I_{C_{1}, f}=\lim _{n \rightarrow \infty} \Phi_{n} ; \quad \Phi_{n} \in \mathrm{Cyl}^{\infty} .
$$

Notice that in fact we have quite a large freedom in a choice of the sequence converging to $I_{C_{1}, f}$; to see this consider a sequence of (analytic) diffeomorphisms $\left(\varphi_{n}\right): \mathbb{R}^{d} \rightarrow \mathbb{R}^{d}$ such that every $\varphi_{n}$ preserves the cube $C_{1}$. Then, applying (6.2) and the unitarity of $u_{\varphi}$ we obtain:

$$
\left\|I_{C_{1}, f}-\Phi_{n}\right\|_{\nu}=\left\|u_{\varphi_{n}}\left(I_{C_{1}, f}-\Phi_{n}\right)\right\|_{\nu}=\left\|I_{C_{1}, f}-u_{\varphi_{n}}\left(\Phi_{n}\right)\right\|_{\nu}
$$


which means that $u_{\varphi_{n}}\left(\Phi_{n}\right)$ converges to $I_{C_{1}, f}$ as well. We will use this freedom to construct a special sequence which converges to $I_{C_{1}, f}$.

Let us fix a number $n$ and consider a graph $\gamma_{n}$ compatible with the cylindrical function $\Phi_{n}$. In general, some edges of the graph can be transversal to the cube $C_{2}$, i.e. they can have isolated intersection points with $C_{2}$. Then the action of $\hat{X}_{C_{2}, f}$ on $\Phi_{n}$ is nontrivial,

$$
\hat{X}_{C_{2}, f} \Phi_{n} \neq 0 \text {. }
$$

Lemma 6.1 For each of the graphs $\gamma_{n}, n=1, \ldots$, defined above, there exists an analytic diffeomorphism $\varphi_{n}: \mathbb{R}^{d} \rightarrow \mathbb{R}^{d}$, such that:

1. $\varphi_{n}$ preserves the cube $C_{1}$;

2. the graph $\varphi_{n}\left(\gamma_{n}\right)$ has no edges transversal to the cube $C_{2}$.

Such a diffeomorphism is constructed explicitly in Appendix.

Use now the diffeomorphisms $\left(\varphi_{n}\right)$ given by Lemma 6.1 to construct the following sequence convergent to $I_{C_{1}, f}$,

$$
\tilde{\Phi}_{n}:=u_{\varphi_{n}}\left(\Phi_{n}\right) \text {. }
$$

Now, each function in the sequence $\left(\tilde{\Phi}_{n}\right)$ is compatible with a graph having no edge transversal to the cube $C_{2}$, hence:

$$
\hat{X}_{C_{2}, f} \tilde{\Phi}_{n}=0 .
$$

\subsubsection{The vanishing of $\left\langle I_{C_{1}, f} \mid I_{C_{2}, f}\right\rangle_{\nu}$}

Equation (3.12) in the case $\Phi^{\prime}=1$ gives

$$
\left\langle\hat{X}_{C_{2}, f} \Phi \mid 1\right\rangle_{\nu}=2\left\langle\Phi \mid I_{C_{2}, f}\right\rangle_{\nu},
$$

for every smooth cylindrical function $\Phi \in L^{2}\left(\overline{\mathcal{A}}, \mu_{\nu}\right)$. Owing to Equation (6.7)

$$
0=\lim _{n \rightarrow \infty}\left\langle\hat{X}_{C_{2}, f} \tilde{\Phi}_{n} \mid 1\right\rangle_{\nu}=\lim _{n \rightarrow \infty} 2\left\langle\tilde{\Phi}_{n} \mid I_{C_{2}, f}\right\rangle_{\nu}=2\left\langle I_{C_{1}, f} \mid I_{C_{2}, f}\right\rangle_{\nu}
$$

This result together with Equation (6.6) imply

$$
\left\|I_{C, f}\right\|_{\nu}^{2}=0 \text {, hence } I_{C, f}=I_{C, f^{\nu}}{ }^{2}=0,
$$

(where the last equality refers to elements of $L^{2}\left(\overline{\mathcal{A}}, \mu_{\nu}\right)$ while the measure $\mu_{\nu}$ is not assumed to be faithful), and completes the first step of the proof. 


\subsection{Step 2: Which measures on $\overline{\mathcal{A}}$ admit a symmetric action of $\hat{X}_{C, f}$ ?}

Equation (3.12) and (6.8) imply, that for any constant function $f$ and for every smooth cylindrical functions $\Phi, \Phi^{\prime}$ :

$$
\left\langle\hat{X}_{C, f} \Phi \mid \Phi^{\prime}\right\rangle_{\nu}-\left\langle\Phi \mid \hat{X}_{C, f} \Phi^{\prime}\right\rangle_{\nu}=0
$$

This means that for every surface $C^{\prime}$ diffeomorphic to the cube (6.5) the operator $\hat{X}_{C^{\prime}, f}$ is symmetric on $\mathrm{Cyl}^{\infty} \subset L^{2}\left(\overline{\mathcal{A}}, \mu_{\nu}\right)$. To complete the proof of (or disprove) Theorem 5.1 we have to answer the question asked in the title of this subsection. The answer is given by the lemma we formulate now, where by a cube, we mean every surface $C^{\prime} \in \mathbb{R}^{d}$ which is diffeomorphic to the cube $C$ considered in Section 6.1.2

Lemma 6.2 Let $G$ be a compact, connected group. Suppose that, for every $(d-1)$-dimensional cube $C$ in $\mathbb{R}^{d}$ and for every constant function $f: C \rightarrow G^{\prime}$ the operator $\hat{X}_{C, f}$ is symmetric on $\mathrm{Cyl}^{\infty} \subset L^{2}(\overline{\mathcal{A}}, \mu)$. Then

$$
\mu=\mu_{\mathrm{AL}} .
$$

The lemma means, that for every $\nu \in \mathcal{N}$ the measure $\mu_{\nu}=\mu_{\mathrm{AL}}$, so the proof of the lemma completes the proof of the first conclusion of the main theorem. On the other hand, for every submanifold $S$ and every function $f$ the operator $\hat{X}_{S, f}$ is self-adjoint on $L^{2}\left(\overline{\mathcal{A}}, \mu_{\mathrm{AL}}\right)$ [7]. Thus the first conclusion and Equation (3.12) imply the second conclusion of the theorem; i.e., that for every $\nu$ the function $F_{S, f}{ }^{\nu}$ is real valued.

\subsection{Proof of Lemma 6.2}

Let us recall, that any regular, Borel, probability measure $\mu$ on the space $\overline{\mathcal{A}}$ is uniquely determined by its projections $\mu_{\gamma}$ on the spaces $\overline{\mathcal{A}}_{\gamma}$ (see Section (4.2) ), where we take into account all possible analytic graphs $\gamma$ embedded in $\mathbb{R}^{d}[12$. Hence, to prove the lemma it is enough to find out what restrictions are imposed by the assumptions of the lemma on each measure $\mu_{\gamma}$.

Every $\mu_{\gamma}$ is a probability measure on the space $\overline{\mathcal{A}}_{\gamma} \cong G^{N}$, where $N$ is the number of edges of a given graph $\gamma$. We outline now the properties of the group $G$ which will be used below. 
As every compact connected Lie group, $G$ is isomorphic [16] to a quotient $\tilde{G} / M$, where $M$ is a central discrete subgroup of $\tilde{G}$, and $\tilde{G}$ is a simple product:

$$
\tilde{G}=T \times P
$$

of an abelian group ${ }^{5} T$ and a semisimple group $P$ (obviously, both $T$ and $P$ are compact and connected). The Lie algebras $\tilde{G}^{\prime}$ and $G^{\prime}$ of $\tilde{G}$ and $G$, respectively, are isomorphic. The left (right) invariant vector fields on $G$ are generated by curves:

$$
[-\epsilon, \epsilon] \ni s \mapsto g \exp (f s)(\exp (f s) g) \in G,
$$

where $\epsilon>0, f \in \tilde{G}^{\prime}$, and $\exp (f s) \in \tilde{G}$.

We emphasize that, in what follows, we will regard $\exp (f s)$ as an element of $\tilde{G}$ rather $G$. Then, the expression

$$
g \exp (f s) \in G
$$

is given by the (natural) action of $\exp (f s) \in \tilde{G}$ on $g \in G$. The advantage is that every $\tilde{g} \in \tilde{G}$ can be expressed as a product of elements of $T$ and $P$.

\subsubsection{Action of $\hat{X}_{C, f}$ on cylindrical functions compatible with a given graph}

We have assumed, that $\hat{X}_{C, f}$ is symmetric on $\mathrm{Cyl}^{\infty} \subset L^{2}(\overline{\mathcal{A}}, \mu)$. Therefore,

$$
0=\left\langle 1 \mid \hat{X}_{C, f} \Psi\right\rangle=\int_{\overline{\mathcal{A}}} \hat{X}_{C, f} \Psi d \mu
$$

for every $\Psi \in \mathrm{Cyl}^{\infty}$. We will fix a graph $\Gamma$ now, and investigate the consequences of the equality (6.9), by using the cubes suitably adjusted to the graph. The adjustment depends on the value of the constant function $f$.

Let us consider an arbitrary graph $\gamma$ consisting of edges $\left\{e_{1}, \ldots, e_{N}\right\}$. Divide each edge $e_{I}$ (by adding a new vertex $v_{I}$ ) into edges $e_{I, i}(i=1,2)$ such that $e_{I}=e_{I, 1} \circ e_{I, 2}$; a graph obtained in this way from the graph $\gamma$ will be denoted by $\Gamma$. Notice that given a measure $\mu$ on $\overline{\mathcal{A}}$, it is enough to consider only all the graphs $\Gamma$ given by the construction above and by all the graphs $\gamma$ in $\Sigma$, to reconstruct $\mu$ from the measures $\mu_{\Gamma}$.

Every cylindrical function $\Psi_{\Gamma}$ compatible with $\Gamma$ can be written in the form:

$$
\Psi_{\Gamma}(\bar{A})=\psi\left(g_{1}(\bar{A}), g_{2}(\bar{A})\right),
$$

\footnotetext{
${ }^{5}$ In fact, $T$ is isomorphic to a tori $U(1)^{n}$ for some $n$.
} 
where:

$$
g_{i}(\bar{A}):=\left(\bar{A}\left(e_{1, i}\right), \ldots, \bar{A}\left(e_{N, i}\right)\right) \in G^{N},
$$

and $\psi$ is a function on $G^{2 N}$.

Now, to draw the conclusions from Equation (6.9), we consider two separate cases: $(i) f \in T^{\prime}$, and $(i i) f \in P^{\prime}$, where $T^{\prime}$ and $P^{\prime}$ are the Lie algebras of the groups $T$ and $P$, respectively. We start with the more complicated case of the semisimple algebra $P^{\prime}$.

6.3.1.1 The case of $f$ valued in $P^{\prime} \quad$ Let $S$ consist of $N$ disjoint cubes $\left\{C_{I}\right\}$, such that each cube $C_{I}$ meets the graph $\Gamma$ only at the vertex $v_{I}$ introduced above (as the division point of an edge $e_{I}$ in the graph $\gamma$ ). Let the orientation of each of the cubes coincide with the orientation of the corresponding edge $e_{I}$. Suppose the function $f$ is defined on $S$ in the following way:

$$
\left.f\right|_{C_{I}}:=\text { const }_{I}=f_{I} \in P^{\prime}
$$

(i.e. we do not assume, that $f_{I}=f_{J}$ for distinct $\left.I, J\right)$. Consider the operator

$$
\hat{X}_{S, f}=\sum_{I=1}^{N} \hat{X}_{C_{I}, f_{I}} .
$$

Assuming that $\Psi_{\Gamma}$ is a smooth cylindrical function, using (6.9) we get

$$
\begin{aligned}
& 0=\int_{\overline{\mathcal{A}}} \hat{X}_{S, f} \Psi_{\Gamma} d \mu=\int_{\overline{\mathcal{A}}_{\Gamma}} \hat{X}_{S, f} \psi d \mu_{\Gamma}= \\
&=-\left.\frac{i}{2} \int_{G^{2 N}} \frac{d}{d s}\right|_{s=0} \psi\left(g_{1} \exp (\vec{f} s), \exp (\vec{f} s) g_{2}\right) d \mu_{\Gamma}= \\
& \quad=-\left.\frac{i}{2} \frac{d}{d s}\right|_{s=0} \int_{G^{2 N}} \psi\left(g_{1} \exp (\vec{f} s), \exp (\vec{f} s) g_{2}\right) d \mu_{\Gamma}
\end{aligned}
$$

where

$$
\begin{aligned}
& \vec{f}:=\left(f_{1}, \ldots, f_{N}\right) \in P^{\prime N} \text { and } \\
& \qquad \exp (\vec{f} s)=\left(\exp \left(f_{1} s\right), \ldots, \exp \left(f_{N} s\right)\right) \in P^{N} .
\end{aligned}
$$

The two latter equations need some comments:

1. Notice that $f$ is valued in $\tilde{G}^{\prime}=T^{\prime} \oplus P^{\prime}$, so in fact expression $\vec{f} \in P^{\prime N}$ means

$$
\vec{f} \in\left\{0_{T}\right\} \times P^{\prime N}
$$


where $0_{T}$ is zero in $T^{N}$. Hence

$$
\exp (\vec{f} s)=\left(\mathbb{I}_{T}, b\right)
$$

where $\mathbb{I}_{T}$ is the neutral element of $T^{N}$ and $b \in P^{N}$. Thus $\exp (\vec{f} s) \in \tilde{G}^{N}$ and the expression $g \exp (\vec{f} s)$ means a natural action of an element of $\tilde{G}^{N}$ on $G^{N}=\tilde{G}^{N} / M^{N}$.

2. In the last step of Equation (6.10) we have changed the order of the integration on $G^{2 N}$ and differentiation with respect to parameter $s$. To justify this we remark that we have integrated a continuously differentiable function over a compact set $G^{2 N}$ (see e.g. [17]).

Equation (6.10) holds for every smooth function on $G^{2 N}$, in particular for

$$
\tilde{\psi}\left(g_{1}, g_{2}\right):=\psi\left(g_{1} \exp \left(\vec{f} s^{\prime}\right), \exp \left(\vec{f} s^{\prime}\right) g_{2}\right) .
$$

Setting $\tilde{\psi}$ into Equation (6.10) we get

$$
0=\frac{d}{d s} \int_{G^{2 N}} \psi\left(g_{1} \exp (\vec{f} s), \exp (\vec{f} s) g_{2}\right) d \mu_{\Gamma},
$$

at an arbitrary value of $s$. The fact that $\exp (\vec{f} s)$ is an element of the connected, compact group $P^{N}$ allows us to write

$$
\int_{G^{2 N}} \psi\left(g_{1} b, b g_{2}\right) d \mu_{\Gamma}=\int_{G^{2 N}} \psi\left(g_{1}, g_{2}\right) d \mu_{\Gamma}
$$

for every $b \in P^{N}$.

Now fix a function $\psi$ and define a map

$$
P^{N} \times P^{N} \ni\left(a, a^{\prime}\right) \mapsto \zeta\left(a, a^{\prime}\right):=\int_{G^{2 N}} \psi\left(g_{1} a, a^{\prime} g_{2}\right) d \mu_{\Gamma} \in \mathbb{C} .
$$

Notice that the function $\zeta$ is differentiable - indeed, it is defined as an integral of a smooth function over a compact set - hence to calculate a derivative of $\zeta$ we can first calculate an appropriate derivative of $\psi\left(g_{1} a, a^{\prime} g_{2}\right)$ and then by integrating the result get the desired derivative of $\zeta$.

Equation (6.12) implies immediately the following property of $\zeta$ :

$$
\zeta\left(b a, a^{\prime} b\right)=\zeta\left(a, a^{\prime}\right)
$$

for every $b \in P^{N}$. Let $b=a^{-1}$. Then

$$
\zeta\left(a, a^{\prime}\right)=\zeta\left(\mathbb{I}_{P}, a^{\prime} a^{-1}\right)=: \xi\left(a^{\prime} a^{-1}\right),
$$


where $\mathbb{I}_{P}$ is the neutral element of $P^{N}$. Now, (6.13) reads

$$
\xi\left(a^{\prime} b a^{-1} b^{-1}\right)=\xi\left(a^{\prime} a^{-1}\right) .
$$

The substitution $b_{0}=a^{\prime} a^{-1}$ gives an identity

$$
\xi\left(b_{0} a b a^{-1} b^{-1}\right)=\xi\left(b_{0}\right),
$$

which holds for every $a, b, b_{0} \in P^{N}$. Notice now, that if $L_{1}, L_{2}$ are arbitrary left invariant vector fields on $P^{N}$, then the vector $\left[L_{1}, L_{2}\right]_{b_{0}}$ tangent to $P^{N}$ at the point $b_{0}$ can be generated by a curve of the form

$$
b_{0} a(t) b(t) a^{-1}(t) b^{-1}(t) .
$$

Thus ${ }^{6}$ :

$$
\left[L_{1}, L_{2}\right]_{b_{0}} \xi=0
$$

But the group $P^{N}$ is semisimple, which means that $\left[P^{\prime N}, P^{\prime N}\right]=P^{\prime N}$. Hence the function $\xi$ and consequently the function $\zeta$ both are constant. This leads us to the following conclusion:

$$
\int_{G^{2 N}} \psi\left(g_{1} b_{1}, b_{2} g_{2}\right) d \mu_{\Gamma}=\int_{G^{2 N}} \psi\left(g_{1}, g_{2}\right) d \mu_{\Gamma}
$$

for every smooth function $\psi$ on $G^{2 N}$ and for every $b_{1}, b_{2} \in P^{N}$.

6.3.1.1 The case of $f$ valued in $T^{\prime}$ Let $S$ consist now of $2 N$ disjoint cubes $C_{I, i}(I=1, \ldots, N$ and $i=1,2)$. Assume, that each $C_{I, i}$ meets the graph $\Gamma$ in exactly one point $y_{I, i} \in e_{I, i}$, such that no $y_{I, i}$ is a vertex of $\Gamma$, and that the orientation of each cube coincides with the orientation of the corresponding edge. Let the function $f$ be

$$
\left.f\right|_{C_{I, i}}=\text { const }_{I, i}=f_{I, i} \in T^{\prime} .
$$

Then corresponding operator is

$$
\hat{X}_{S, f}=\sum_{i=1,2} \sum_{I=1}^{N} \hat{X}_{C_{I, i}, f_{I, i}} .
$$

A derivation ${ }^{7}$ similar to that one shown in the previous paragraph gives us the following counterpart of Equation (6.14):

$$
\int_{G^{2 N}} \psi\left(g_{1} t_{1}, t_{2} g_{2}\right) d \mu_{\Gamma}=\int_{G^{2 N}} \psi\left(g_{1}, g_{2}\right) d \mu_{\Gamma}
$$

for every $t_{1}, t_{2} \in T^{N}$.

\footnotetext{
${ }^{6}$ Recall that $\zeta$ is differentiable, thus the function $\xi$ is differentiable as well.

${ }^{7}$ In general the operator $\hat{X}_{S, f}$ is a sum of right and left invariant vector fields on a product of some copies of $G$. But the abelian subgroup $T$ is a central subgroup of
} 


\subsubsection{Final conclusion}

Let us now combine the results (6.14) and 6.15). Because $T^{N}$ is a central subgroup of $T^{N} \times P^{N}$, every $t \in T^{N}$ commutes with every $b \in P^{N}$. This gives us:

$$
\int_{G^{2 N}} \psi\left(g_{1} t_{1} b_{1}, t_{2} b_{2} g_{2}\right) d \mu_{\Gamma}=\int_{G^{2 N}} \psi\left(g_{1}, g_{2}\right) d \mu_{\Gamma}
$$

for every $t_{i} b_{i} \in T^{N} \times P^{N}$, that is:

$$
\int_{G^{2 N}} \psi\left(g_{1} h_{1}, h_{2} g_{2}\right) d \mu_{\Gamma}=\int_{G^{2 N}} \psi\left(g_{1}, g_{2}\right) d \mu_{\Gamma}
$$

for every $h_{i} \in \tilde{G}^{N}$ (or equivalently for every $h_{i} \in G^{N}$ ) and for every smooth function $\psi$ on $G^{2 N}$. By virtue of the Stone-Weierstrass theorem the closure in the sup-norm of the space $C^{\infty}\left(G^{2 N}, \mathbb{C}\right)$ is $C^{0}\left(G^{2 N}, \mathbb{C}\right)$, hence Equation (6.16) holds for every $\psi \in C^{0}\left(G^{2 N}, \mathbb{C}\right)$.

To show that $\mu_{\Gamma}$ is the Haar measure on $G^{2 N}$ let us define the following map on $G^{2 N}$ :

$$
\left(G^{N}, G^{N}\right) \ni\left(g_{1}, g_{2}\right) \mapsto \omega\left(g_{1}, g_{2}\right):=\left(g_{1}, g_{2}^{-1}\right) \in\left(G^{N}, G^{N}\right) .
$$

Then (6.16) implies that the push forward measure

$$
\mu_{\Gamma}^{*}:=\omega^{*} \mu_{\Gamma}
$$

is the right invariant Haar measure on $G^{2 N}$. Indeed, a direct calculation gives for every $\left(h_{1}, h_{2}\right) \in G^{N} \times G^{N}$

$$
\int_{G^{2 N}} \psi\left(g_{1}, g_{2}\right) d \mu_{\Gamma}^{*}=\int_{G^{2 N}} \psi\left(g_{1} h_{1}, g_{2} h_{2}^{-1}\right) d \mu_{\Gamma}^{*}
$$

Since $G$ is compact, the Haar measure $\mu_{\Gamma}^{*}$ is invariant with respect to the map $\omega$, hence $\mu_{\Gamma}$ is the Haar measure itself ${ }^{8}$.

$T \times P=\tilde{G}$ and the elements of $T$ commute with all the elements of $\tilde{G}$ (and of $G$ ). This means, that if $f$ is valued in $T^{\prime}$, then corresponding left and right invariant vector fields defining the action of $\hat{X}_{S, f}$ coincide and:

$$
\hat{X}_{S, f} \Psi_{\Gamma}=-\left.i \frac{d}{d s}\right|_{s=0} \psi\left(g_{1} \exp \left(\vec{f}_{1} s\right), \exp \left(\vec{f}_{2} s\right) g_{2}\right),
$$

where $\vec{f}_{i}=\left(f_{1, i}, \ldots, f_{N, i}\right) \in T^{N}$. Obviously we have $\exp \left(\vec{f}_{i} s\right) g_{i}=g_{i} \exp \left(\vec{f}_{i} s\right)$.

${ }^{8}$ The Haar measure $\mu_{\Gamma}^{*}$ on $G^{2 N}$ is a product of two copies of the Haar measure $\mu_{H}$ on $G^{N}$. On the other hand for every compact group left and right invariant Haar measures on the group coincide 18. Applying the latter fact to one of the $\mu_{H}$ 's in the product constituting $\mu_{\Gamma}^{*}$ we get the invariance of $\mu_{\Gamma}^{*}$ with respect to $\omega$. Thus $\mu_{\Gamma}^{*}$ is left and right invariant Haar measure on $G^{2 N}$. 
Every graph $\Gamma$ is obtained by a subdivision of some graph $\gamma$, hence every cylindrical function $\Psi$ compatible with $\gamma$ is also compatible ${ }^{9}$ with $\Gamma$. Thus making use of Equation (4.3) we conclude, that the measure $\mu_{\gamma}$ on $\overline{\mathcal{A}}_{\gamma} \cong G^{N}$ is the Haar measure. Because the graph $\gamma$ is arbitrary,

$$
\mu=\mu_{\mathrm{AL}} .
$$

This completes the proof of lemma 6.2 and the proof of the main theorem.

\section{$7 \quad$ Summary}

In this paper we considered diffeomorphism covariant $*$-representations of the Sahlmann holonomy-flux algebra $\mathfrak{A}$ (Definition 3.1. Definition 4.1) for a $G$-connection theory, where $G$ is an arbitrary compact connected Lie group. We showed that if the principle bundle underlying the algebra is a trivial bundle $^{10} \Sigma \times G$ and if $\Sigma=\mathbb{R}^{d}$, then the carrier space used in Sahlmann's Characterization 3.2 is the orthogonal product (Theorem [5.1):

$$
\mathcal{H}=\bigoplus_{\nu} L^{2}\left(\overline{\mathcal{A}}, \mu_{\nu}\right)
$$

where every measure $\mu_{\nu}$ is the natural measure:

$$
\mu_{\nu}=\mu_{\mathrm{AL}}
$$

We emphasize that although the requirement of the diffeomorphism covariance of the representation singles out the measure $\mu_{\mathrm{AL}}$ there may possibly exist inequivalent diffeomorphism covariant representations of the Sahlmann algebra on the Hilbert space (7.1), which differ from each other in the family of functions $\left\{F_{S, f^{\iota} \nu}\right\}$ (notice that the functions are not completely determined by Theorem [5.1). Thus the covariance with respect to the group of analytic diffeomorphisms does not seem to fix a unique diffemorphism covariant representation. However, an action of a larger group of diffeomorphisms (including certain non-analytic ones) is naturally defined on the algebra; the covariance with respect to that group singles out precisely one (irreducible) representation of the Sahlmann algebra [20].

\footnotetext{
${ }^{9}$ Notice that $\Psi(\bar{A})=\psi\left(\bar{A}\left(e_{I}\right)\right)=\psi\left(\bar{A}\left(e_{I, 1}\right) \bar{A}\left(e_{I, 2}\right)\right)$.

${ }^{10}$ The result can be obtained in the general case of an arbitrary bundle $P(\Sigma, G)$, where $\Sigma$ is an arbitrary real-analytic manifold and $G$ is a compact connected Lie group [19].
} 
An independent side result of our work is a proof showing that, if a given measure $\mu$ on $\overline{\mathcal{A}}$, the operators $\left\{\hat{X}_{S, f}\right\}$ are symmetric in $\mathrm{Cyl}^{\infty} \subset L^{2}(\overline{\mathcal{A}}, \mu)$, for some sufficiently large class of submanifolds $S \subset \Sigma$ and constant functions $f: S \rightarrow G^{\prime}$, then $\mu=\mu_{\mathrm{AL}}$ (Lemma 6.2). This means that for every manifold $\Sigma$ the $*$-representation $\pi$ of $\mathfrak{A}$ defined on $\mathcal{H}=L^{2}(\overline{\mathcal{A}}, \mu)$ as the identity i.e., the representation

$$
\pi(\Phi) \Psi:=\Phi \Psi \quad \pi\left(\hat{X}_{S, f}\right) \Psi:=\hat{X}_{S, f} \Psi
$$

requires (7.2).

Another observation we made is that every $*$-representation of the subalgebra $\mathrm{Cyl}^{\infty} \subset \mathrm{Cyl}$ can be uniquely extended to a $*$-representation of the Ashtekar-Isham $C^{\star}$-algebra $\mathrm{Cyl}$ (this fact enabled us to define the algebra $\mathfrak{A}$ by means of derivatives $\mathcal{X}$ and only smooth cylindrical functions).

Acknowledgements: Our interest in this subject was stimulated by discussions with Hanno Sahlmann who also explained to us many technical points and discussed with us his unpublished considerations. Stanisław L. Woronowicz and Jacek Tafel gave us very good hints helpful in proving Lemma 3.1 and Lemma 6.2 respectively. Finally, we thank Abhay Ashtekar and Thomas Thiemann for numerus discussions about the quantum geometry and, respectively, QSD. This work was supported in part by the following Polish KBN grants: 2 PO3B 068 23, 2 PO3B 12724.

\section{A Construction of the diffeomorphism used in Le- mma 6.1}

In proving the main theorem we left lemma 6.1 without any proof. Let us now fill that gap. The lemma states that:

Lemma A.1 For each graph $\gamma$ there exists an analytic diffeomorphism $\varphi$ : $\mathbb{R}^{d} \rightarrow \mathbb{R}^{d}$, such that:

1. $\varphi$ preserves the cube $C_{1}$;

2. the graph $\varphi(\gamma)$ has no edges transversal to the cube $C_{2}$.

Proof. Let us recall definitions of cubes $C_{i}(i=1,2)$ :

$$
\begin{aligned}
& C_{1}:=\left\{y \in C \mid x^{1}(y) \leq 0\right\} \\
& C_{2}:=\left\{y \in C \mid x^{1}(y)>0\right\},
\end{aligned}
$$


where

$$
C:=\left\{y \in \mathbb{R}^{d} \mid\left\{\begin{array}{l}
x^{d}(y)=0 \\
-l<x^{1}(y) \leq l \\
-l<x^{i}(y)<l \quad \text { for } i=2,3, \ldots, d-1 .
\end{array}\right.\right.
$$

Here $\left(x^{j}\right),(j=1, \ldots, d)$ is a coordinate frame on $\mathbb{R}^{d}$ obtained by an affine transformation of the Cartesian coordinates on $\mathbb{R}^{d}$.

Assume that the set $\left\{\tilde{e}_{J}\right\}$ contains all edges of the graph $\gamma$ such that each $\tilde{e}_{J}$ intersects the cube $C_{2}$ in isolated points. Because the set $\left\{\tilde{e}_{J}\right\}$ is finite and the edges and cube $C_{2}$ are analytic, the set $W:=\left(\bigcup_{J} \tilde{e}_{J}\right) \cap C_{2} \subset \mathbb{R}^{d}$ (i.e. the set of intersection points of the cube and the edges) is finite as well. Define

$$
\epsilon:=\min \left\{x^{1}(y) \mid y \in W\right\} .
$$

Clearly $\epsilon>0$. Let us assume now that the function $\varphi^{\prime}: \mathbb{R} \rightarrow \mathbb{R}$ is an analytic diffeomorphism such that:

$$
\left\{\begin{array}{l}
\varphi^{\prime}(-l)=-l \\
\varphi^{\prime}(0)=0 \\
\varphi^{\prime}(\epsilon)>l
\end{array}\right.
$$

Then we can define

$$
\varphi^{i}\left(x^{j}\right):= \begin{cases}\varphi^{\prime}\left(x^{1}\right) & \text { for } i=1 \\ x^{i} & \text { otherwise. }\end{cases}
$$

In this way we have reduced our task to constructing the diffeomorphism $\varphi^{\prime}$.

To construct the diffeomorphism it will be convenient to transform the coordinate $x^{1}$ in the following way:

$$
\left.\mathbb{R} \ni x^{1} \mapsto z\left(x^{1}\right):=\exp \left[\frac{\ln 2}{l} x^{1}\right] \in\right] 0, \infty[.
$$

Values of the new coordinate at points relevant for us are the following:

$$
\begin{gathered}
z(-l)=\frac{1}{2}, \quad z(l)=2, \\
z(0)=1, \quad z(\epsilon)=1+\epsilon^{\prime}
\end{gathered}
$$


for some $\epsilon^{\prime}>0$. Consider now two families of analytic diffeomorphisms on ] $0, \infty[$

$$
\begin{aligned}
\alpha_{t}(z) & :=\frac{(1+t)^{z}-1}{2 t} \\
\beta_{s}(z) & :=\frac{\ln (1+s z)}{2 \ln (1+s)},
\end{aligned}
$$

where $t, s \in] 0, \infty[$. We have

$$
\begin{gathered}
\lim _{t \rightarrow \infty} \alpha_{t}(z)= \begin{cases}\infty & \text { for } z>1 \\
\frac{1}{2} & \text { for } z=1 \\
0 & \text { for } z<1\end{cases} \\
\lim _{s \rightarrow \infty} \beta_{s}(z)=\frac{1}{2} ; \quad \lim _{s \rightarrow 0} \beta_{s}(z)=\frac{1}{2} z .
\end{gathered}
$$

Equation (A.2) means that it is possible to find $t_{0}$, such that:

$$
\alpha_{t_{0}}\left(1+\epsilon^{\prime}\right)>2
$$

and:

$$
\alpha_{t_{0}}\left(\frac{1}{2}\right)<\frac{1}{4}
$$

From (A.3) we know that for some $s_{0}$

$$
\beta_{s_{0}}\left(\frac{1}{2}\right)=\frac{1}{2}-\alpha_{t_{0}}\left(\frac{1}{2}\right) .
$$

Then

$$
\phi(z):=\alpha_{t_{0}}(z)+\beta_{s_{0}}(z)
$$

is an analytic diffeomorphism on $] 0, \infty[$ such that:

$$
\left\{\begin{array}{l}
\phi\left(\frac{1}{2}\right)=\frac{1}{2} \\
\phi(1)=1 \\
\phi\left(1+\epsilon^{\prime}\right)>2
\end{array},\right.
$$

which coincides with (A.1). Thus

$$
\varphi^{\prime}\left(x^{1}\right):=\frac{l}{\ln 2} \ln \left[\phi\left(z\left(x^{1}\right)\right)\right] .
$$




\section{References}

[1] Sahlmann H 2002 Some Comments on Representation Theory of the Algebra Underlying Loop Quantum Gravity Preprint gr-qc/0207111

[2] Sahlmann H 2002 When Do Measures on the Space of Connections Support the Triad Operators of Loop Quantum Gravity? Preprint gr-qc/0207112

[3] Sahlmann H and Thiemann T 2003 On the Superselection Theory of the Weyl Algebra for Diffeomorphism Invariant Quantum Gauge Theories Preprint gr-qc/0302090

[4] Ashtekar A and Lewandowski J 1995 Differential geometry on the space of connections using projective techniques, Jour. Geo. \& Phys. 17, 191230

[5] Rovelli C and Smolin L 1995 Discreteness of area and volume in quantum gravity Nucl. Phys. B442 593-622 Erratum: Nucl. Phys. B456 753 Preprint gr-qc/9411005

Loll R 1996 The volume operator in discretized quantum gravity Phys. Rev. Lett. 75 3048-3051 Preprint gr-qc/9506014

Thiemann T 1998 Closed formula for the matrix elements of the volume operator in canonical quantum gravity J. Math. Phys. 39 3347-3371 Preprint gr-qc/9606091

[6] Thiemann T 1998 A length operator for canonical quantum gravity $J$. Math. Phys. 39 3372-3392 Preprint gr-qc/9606092

[7] Ashtekar A and Lewandowski J 1997 Quantum theory of geometry. I: Area operators Class. Quant. Grav. 14 A55-A82 Preprint gr-qc/9602046

[8] Ashtekar A and Lewandowski J 1998 Quantum Theory of Geometry. 2. Volume operators Adv. Theor. Math. Phys. 1 388-429 Preprint gr-qc/9711031

[9] Ashtekar A, Corichi A and Zapata J A 1998 Quantum Theory of Geometry III: Non-commutativity of Riemannian Structures Class. Quant. Grav. 15 2955-2972 Preprint gr-qc/9806041

[10] Rovelli C and Smolin L 1990 Loop representation for quantum general relativity Nucl. Phys. B331 80-152 
Rovelli C and Smolin L 1994 The physical Hamiltonian in nonperturbative quantum gravity Phys. Rev. Lett. 72 446-449 Preprint gr-qc/9308002 Ashtekar A, Lewandowski J, Marolf D, Mourão J and Thiemann T 1995 Quantization of diffeomorphism invariant theories of connections with local degrees of freedom J. Math. Phys. 36 6456-6493, Preprint gr-qc/9504018

Rovelli C 1998 Loop Quantum Gravity Living Rev. Rel. 11 Preprint gr-qc/9710008

[11] Thiemann T 1996 Anomaly-free formulation of non-perturbative, fourdimensional Lorentzian quantum gravity Phys. Lett. B380 257-264 Preprint gr-qc/9606088

Thiemann T 1998 Quantum Spin Dynamics (QSD) Class. Quant. Grav. 15 839-873 Preprint gr-qc/9606089

Thiemann T 1998 QSD III : Quantum Constraint Algebra and Physical Scalar Product in Quantum General Relativity Class. Quant. Grav. 15 1207-1247 Preprint gr-qc/9705017

Thiemann T 1998 QSD V : Quantum Gravity as the Natural Regulator of Matter Quantum Field Theories Class. Quant. Grav. 15 1281-1314 gr-qc/9705019

[12] Ashtekar A and Lewandowski J 1994 Representation theory of analytic holonomy $C^{\star}$-algebras Knots and quantum gravity (Baez J (ed), Oxford: Oxford University Press) Preprint gr-qc/9311010

Marolf D and Mourão J 1995 On the support of the Ashtekar-Lewandowski measure Commun. Math. Phys. 170, 583-606.

Ashtekar A and Lewandowski J 1995 Projective techniques and functional integration for gauge theories J. Math. Phys. 36 2170-2191 Preprint gr-qc/9411046

[13] Baez J C 1994 Generalized measures in gauge theory Lett. Math. Phys. 31 213-223 Preprint hep-th/9310201

[14] Ashtekar A and Isham C J 1992 Representations of the holonomy algebras for gravity and non-Abelian gauge theories Class. Quant. Grav. 9 $1433-1467$

[15] Lewandowski J and Marolf D Loop constraints: A habitat and their algebra, Int. J. Mod. Phys. D7 299-330 Preprint gr-qc/9710016

Baez J C and Sawin S 1997 Functional integration on spaces of connections Jour. Funct. Analysis 150 1-27 Preprint q-alg/9507023

Lewandowski J and Thiemann T 1999 Diffeomorphism invariant quantum 
field theories of connections in terms of webs Class. Quant. Grav. 162299 2322 Preprint gr-qc/9901015

[16] Wojtyński W 1986 Grupy i algebry Liego (Lie groups and algebras) (Warszawa: PWN — Polish Scientific Publisher)

[17] Billingsley P 1979 Probability and measure (New York-ChichesterBrisbane-Toronto: John Wiley \& Sons)

[18] Barut A O and Rạczka R 1977 Theory of group representations and applications (Warszawa: PWN - Polish Scientific Publisher)

[19] Okołów A and Lewandowski L Authomorphism covariant representations of the holonomy-flux $*$-algebra, in preparation

[20] Lewandowski J, Okołów A, Sahlmann H and Thiemann T, in preparation 